\title{
Considerations on compensation of beam-beam effects in the Tevatron with electron beams
}

\author{
V. Shiltsev, V. Danilov,* D. Finley, and A. Sery ${ }^{\dagger}$ \\ Fermi National Accelerator Laboratory, Batavia, Illinois 60510
}

(Received 8 October 1998; published 28 July 1999)

\begin{abstract}
The beam-beam interaction in the Tevatron collider sets limits on bunch intensity and luminosity. These limits are caused by a tune spread in each bunch which is mostly due to head-on collisions, but there is also a bunch-to-bunch tune spread due to parasitic collisions in multibunch operation. We propose to compensate these effects with the use of a countertraveling electron beam, and we present general considerations and physics limitations of this technique.
\end{abstract}

PACS numbers: 41.75.Lx, 29.27.Bd, 41.85.Ew

\section{INTRODUCTION}

Two major Tevatron upgrade projects are under realization and consideration now. One is based on the operation of the Main Injector and the Antiproton Recycler and is called Run II, and the second is called "TEV33." Table I [1,2] gives the main parameters of these upgrades.

The beam-beam interaction between protons and antiprotons takes place at the two head-on interaction points (IPs, located at B0 and D0 sectors), as well as at numerous parasitic crossings where the beam orbits are separated by about a dozen of their rms sizes. Since the proton beam intensity is several times the antiproton intensity, the beam-beam effects are more severe for antiprotons $(\bar{p})$. It is to be noted that the design value of the total tune shift for antiprotons is about the maximum experimentally achieved value for proton colliders $\Delta \nu \approx 0.025$ [3]. The "footprint area" (spread of betatron frequencies) of the $\bar{p}$ beam with such a tune shift is large enough to also cause an increase of particle losses due to higher order lattice resonances $[4,5]$.

In order to achieve sufficient beam-beam separation away from the IPs, a crossing angle of about 200 microradians between proton and antiproton orbits at the main interaction points can be used. Besides the geometrical luminosity reduction, the crossing angle may lead to synchrobetatron coupling, additional resonances, beam blow-up, and luminosity degradation [6], although the maximum tune shift becomes smaller with the angle.

Tevatron beam injection requires some gaps in the bunch train that results in the so-called "PACMAN effect"- bunch-to-bunch variation of the betatron tunes due to long-range beam-beam interactions.

The effect depends on the orbit separation around the ring and is most visible for bunches close to the gaps. For example, the larger circles in Fig. 1 from [2] shows the

\footnotetext{
*Presently at Oak Ridge National Laboratory, Oak Ridge, TN 37831-8218.

†On leave from Budker INP, Novosibirsk, 630090 Russia.
}

spread in vertical and horizontal tunes for small betatron amplitude particles in all $\bar{p}$ bunches for TEV33 with 140 proton and 121 antiproton colliding bunches (see parameters in Table I). In the same figure, the smaller circles represent the tunes of nonzero amplitude particles in three of the $\bar{p}$ bunches. One can see that the tune spread within each bunch and the bunch-to-bunch tune spread are both about 0.008 .

During Run II with 36 bunches in each beam, the bunch-to-bunch spread is expected to be about $\delta \nu \approx$ 0.007 , while the single bunch tune spread will be about $\Delta \nu \approx 0.018$.

Another effect of the long-range interactions is the bunch-to-bunch variation of $x-y$ coupling due to the skew component of the beam-beam kick [2]. That is of concern because the Tevatron operates near the difference resonance $\nu_{x}=20.585, \nu_{y}=20.575$. These tune spreads and the estimated skew-kicks are expected to be a problem for the collider operation if uncorrected.

In this article we consider a technique for compensation of the beam-beam effects in the Tevatron with use of high current, low energy electron beam [7,8]. The electron beam setup (schematically depicted in Fig. 2) is

TABLE I. The Tevatron upgrades.

\begin{tabular}{lcc}
\hline \hline \multicolumn{1}{c}{ Parameter } & Run II & TEV33 \\
\hline Beam energy $E_{b}(\mathrm{GeV})$ & 1000 & 1000 \\
Luminosity $\mathcal{L}\left(\mathrm{s}^{-1} \mathrm{~cm}^{-2}\right)$ & $2.1 \times 10^{32}$ & $1.2 \times 10^{33}$ \\
No. of bunches $(p, \bar{p}) N_{b}$ & 36,36 & 140,121 \\
Min. bunch spacing $\tau(\mathrm{ns})$ & 396 & 132 \\
Protons $/$ bunch $\left(N_{p} / 10^{11}\right)$ & 2.7 & 2.7 \\
Antiprotons $/$ bunch $\left(N_{\bar{p}} / 10^{11}\right)$ & 0.75 & 0.6 \\
$p$ emittance rms $\varepsilon_{n p}(\pi \mu \mathrm{m} \mathrm{rad})$ & 3.3 & 3.3 \\
$\bar{p}$ emittance rms $\varepsilon_{n \bar{p}}(\pi \mu \mathrm{m} \mathrm{rad})$ & 2.5 & 2.5 \\
Number of IPs $N_{\mathrm{IP}}$ & 2 & 2 \\
Interaction focus $\beta^{*}(\mathrm{~cm})$ & 37 & 37 \\
Crossing half-angle $\theta_{\mathrm{IP}}(\mathrm{mrad})$ & 0 & 0.14 \\
Bunch length $\sigma_{s}(\mathrm{~cm})$ & 37 & $37 \rightarrow 14$ \\
$\bar{p}$ tune shift $\Delta \nu_{\bar{p}}$ & $\sim 0.020$ & $\sim 0.015$ \\
$p$ tune shift $\Delta \nu_{p}$ & 0.005 & 0.007 \\
$\bar{p}$ bunch-to-bunch tune spread & 0.007 & 0.010 \\
\hline \hline
\end{tabular}




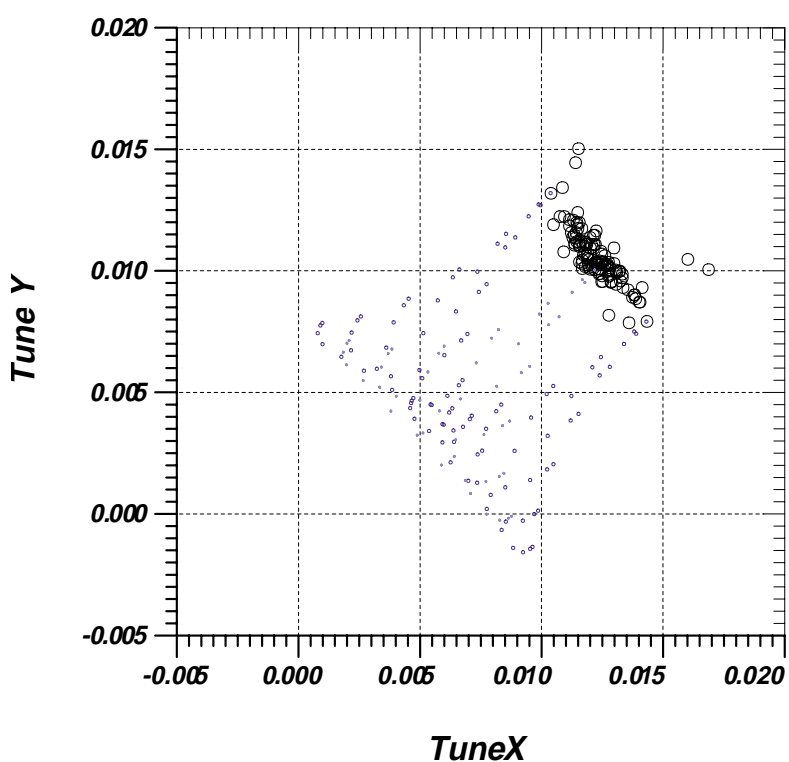

FIG. 1. (Color) Tune spread in the antiproton beam for TEV33. Large circles are for tunes of core particles in 121 antiproton bunches. Small circles are tunes of nonzero betatron amplitude particles in some bunches.

to be installed away from the proton-antiproton interaction points at $\mathrm{B} 0$ and $\mathrm{D} 0$. It provides the electron beam which collides with the antiproton beam. The electron beam is to be born on an electron gun cathode, transported through the interaction section in a strong solenoidal magnetic field, and absorbed in the collector. In principle, since the electron charge is opposite to the proton charge, the electromagnetic force on antiprotons due to the proton beam can be compensated by the electron beam. For the design we consider, the proton beam has to be separated from the electron and antiproton beams in the device.

In Sec. II we consider two implementations of the proposal: (i) an "electron lens" with modulated current to provide different linear defocusing forces for different antiproton bunches in order to equalize their betatron frequencies and (ii) an "electron compressor," that is a nonlinear dc electron lens which compensates (on average) the nonlinear focusing due to the proton beam. Section III is devoted to an analysis of the parameters and stability of the electron beam for the beam-beam compensation. Important side effects due to the electron

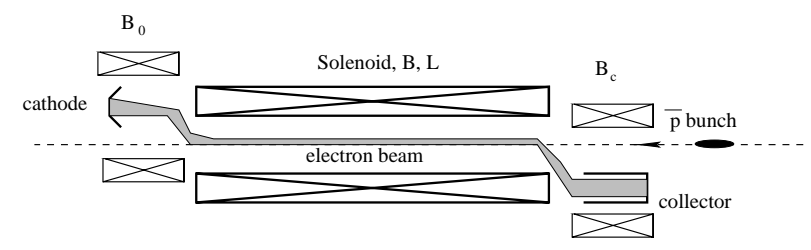

FIG. 2. Layout of the beam-beam compensation device. beam are studied in Sec. IV. In Sec. V we present a historical overview of the beam-beam compensation ideas, discuss applicability of the technique to other colliders, and make conclusions on our work.

Meanings of mathematical symbols used in the paper are listed below.

$\begin{array}{cl}z=(x \text { or } y) & \begin{array}{l}\text { either horizontal or vertical coordinate } \\ \beta^{*}\end{array} \\ \beta_{z} & \begin{array}{l}\text { beta function at IP } \\ \text { beta function at the electron beam } \\ \beta_{e}=v_{e} / c\end{array} \\ \gamma_{e}=\frac{1}{\sqrt{1-\beta_{e}^{2}}} & \text { electron velocity } \\ \gamma_{(p, \bar{p})} & (p, \bar{p}) \text { relativistic factor } \\ \sigma_{s} & \bar{p} \text { rms bunch length } \\ \sigma_{z}=\frac{\sqrt{\varepsilon_{n, z} \beta_{z}}}{\gamma_{\bar{p}}} & \bar{p} \text { rms beam size } \\ a & \text { radius of round electron beam } \\ \sigma_{e} & \text { rms transverse electron beam size } \\ \rho_{(p, \bar{p}, e)}(x, y) & (p, \bar{p}, e) \text { charge distribution } \\ n_{(e, i)} & \text { (electron, ion) density } \\ J & \text { electron current } \\ j_{e}=e n_{e} v_{e} & \text { electron current density } \\ L & \text { electron beam length } \\ \Delta \nu_{z} & \bar{p} \text { tune shift } \\ \Delta \nu_{z}^{p} & p \text { tune shift } \\ \nu_{x}-\nu_{y} \mid & \text { difference of hor.-vert. } \bar{p} \text { tunes } \\ \xi_{z}^{e} & \bar{p} \text { tune shift due to electrons }\end{array}$

\section{BEAM-BEAM COMPENSATION TECHNIQUES}

\section{A. Linear electron lens}

We start with the electron beam lens for compensation of the bunch-to-bunch tune spread in the Tevatron antiproton beam. Time-modulated current of an electron beam can produce defocusing forces necessary to compensate effects caused by parasitic beam-beam interactions with the proton beam. We estimate the main parameters of the electron beam and consider the resulting beam footprint.

For the tune shift of the antiproton bunch due to the electron beam, we calculate the tune shift of a particle with zero horizontal and vertical betatron amplitudes and zero energy offset. The beam-beam interaction can be described in terms of the two-dimensional potential $V(x, y)$, such that the beam-beam kicks given to a particle on each turn are equal to $\Delta z^{\prime}=-\partial V / \partial z$, where $z$ may stand for either $x$ or $y$. The tune shifts for zero amplitude $\bar{p}$ particles which collide head-on with the electron beam can be found from

$$
\Delta \nu_{z}=-\frac{\beta_{z}}{4 \pi} \frac{\partial^{2} V}{\partial z^{2}}
$$


For a round, constant density electron beam with total current $J$, radius $a$, and interacting with antiprotons over length $L$, the potential is axisymmetric and equal to

$$
V(r)=r^{2} \frac{\left(1+\beta_{e}\right) J L r_{\bar{p}}}{e \beta_{e} c a^{2} \gamma_{\bar{p}}}, \quad r^{2}=x^{2}+y^{2},
$$

and the tune shifts are equal to

$$
\xi_{z}^{e}=-\frac{\beta_{z}}{2 \pi} \frac{\left(1+\beta_{e}\right) J L r_{\bar{p}}}{e \beta_{e} c a^{2} \gamma_{\bar{p}}}
$$

where $r_{\bar{p}} \approx 1.53 \times 10^{-18} \mathrm{~m}$ is the (anti)proton classical radius. For example, one needs an electron beam with about $J=1.65$ A of current along a $L=2 \mathrm{~m}$ length, with $a=1 \mathrm{~mm}$ radius, and energy $10 \mathrm{kV}\left(\beta_{e}=0.2\right)$ in order to obtain $\xi^{e} \approx-0.01$ in the Tevatron collider with parameters $\gamma_{p} \approx 1066, \beta_{z}=100 \mathrm{~m}$. If the electron beam radius $a$ is several times the $\bar{p}$ rms beam size $\sigma_{z}$, then most antiprotons have nearly equal tune shifts. The variable in time electron current can be used for the compensation of the bunch-to-bunch tune spread.

Equation (3) shows that both horizontal and vertical tune shifts $\xi_{x}^{e}, \xi_{y}^{e}$ due to head-on collision with electrons have the same (negative) sign. In contrast, long-range beambeam proton-antiproton interaction at parasitic crossings shift horizontal and vertical tunes in opposite directions $\Delta \nu_{x} \approx-\Delta \nu_{y}$. The resulting bunch-to-bunch tune spread along the line $\Delta \nu_{x}+\Delta \nu_{y}$ is several times the spread along $\Delta \nu_{x}-\Delta \nu_{y}$ as seen in Fig. 1.

Obviously, two electron lenses - one at a location with the horizontal beta function larger than vertical $\beta_{x} \gg$ $\beta_{y}$, and another one at $\beta_{x} \ll \beta_{y}$ (see Fig. 3) - can compensate any bunch-to-bunch tune spread. The first one will produce a larger tune shift in the horizontal plane, and the second in the vertical plane.

For our numerical simulations we chose two locations in the Tevatron for two electron lens devices - one at the short straight location called F48, and the other at the up-

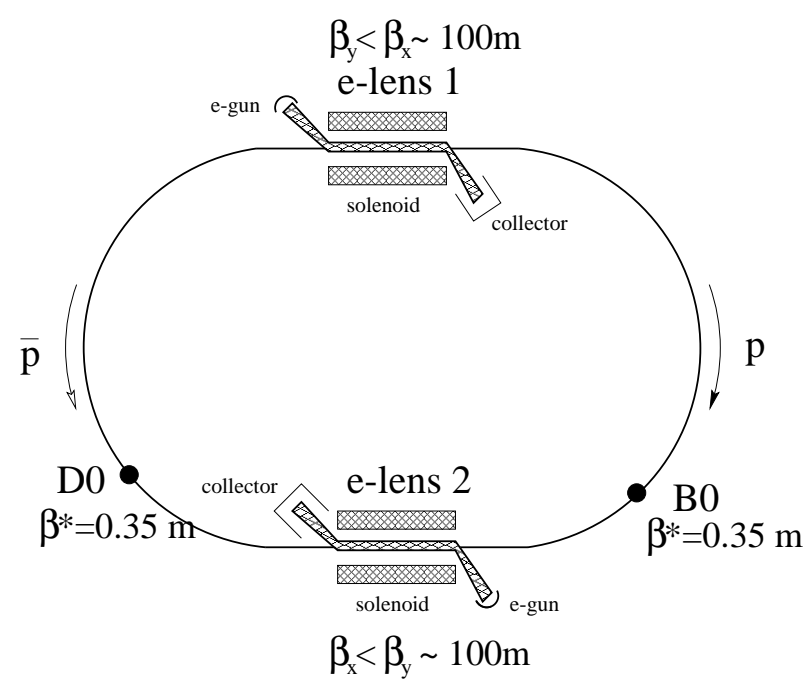

FIG. 3. Schematic Tevatron layout with two electron lenses. stream end of the $\mathrm{C} 0$ straight section. Parameters of these locations and the two corresponding electron beams are presented in Table II. We assume round constant density electron beams with radius about twice the maximum of the horizontal or vertical $\bar{p}$ sizes at the corresponding location $\sigma_{x, y} \simeq 0.5-0.7 \mathrm{~mm}$. Let us demonstrate the compensation technique for the bunch-to-bunch tune spread shown in Fig. 1.

If we denote the currents in the two electron lenses as $J_{1}(t)$ and $J_{2}(t)$, then the core particles' tune shifts due to electrons are equal to

$$
\xi_{z}^{e}(t)=\beta_{1, z} J_{1}(t) C_{1}+\beta_{2, z} J_{2}(t) C_{2} .
$$

The constants are $C_{1,2} \approx \frac{3.03 \times 10^{-5} L[\mathrm{~m}]}{a_{1,2}^{2}[\mathrm{~mm}]}$ for $\beta_{e}=0.2$.

Full compensation of the tune spread requires the currents to be solutions of two linear equations $\xi_{x, y}^{e}(t)=$ $-\Delta \nu_{x, y}(i)$, where $i$ enumerates the bunch number and, therefore, $t=\tau i$. The currents $J_{1}(t)$ and $J_{2}(t)$ vs time $t$ for the TEV33 operation scenario with $140 p$ bunches and $121 \bar{p}$ bunches are shown in Fig. 4. The patterns of these currents have to be repeated periodically with the Tevatron revolution period of about $21 \mu \mathrm{s}$. Positions of all antiproton bunches are marked by circles in Fig. 4 . Minimum bunch spacing is $\tau=132 \mathrm{~ns}$. Two gaps between bunch trains are seen, too; thus, the total number of bunches $N_{b}=121$ is less than $159=21 \mu \mathrm{s} / 132 \mathrm{~ns}$ (if every seventh bucket is filled in the Tevatron). The maximum current is about $2.2 \mathrm{~A}$. The currents $J_{1}, J_{2}$ have complicated waveforms needed to compensate the tune shifts seen in Fig. 1. The result of implementing these lenses would be that all core particle tunes of all the bunches would become identical.

Figure 5 shows the initial 121 bunch tunes and the resulting bunch tunes assuming a 10\% compensation error (see circles in the lower left-hand corner). Such an error may be due to current mismatch, inadequate beam-beam model, or imprecision of the single bunch tune diagnostics. Again, without errors, the result of compensation would look like a point in Fig. 5.

Linear electron lenses with an electron beam radius wider than the rms $\bar{p}$ size almost do not distort the footprint of each bunch [8], and, therefore, the compensation of the bunch-to-bunch tune spread only will give about a twofold reduction of the tune area covered by the Tevatron $\bar{p}$ beam.

TABLE II. Bunch-to-bunch electron beam lenses.

\begin{tabular}{lcc}
\hline \hline \multicolumn{1}{c}{ Parameter } & Location 1 & Location 2 \\
\hline & & \\
Horiz. beta function $\beta_{1,2}^{x}(\mathrm{~m})$ & 101.7 & 59.0 \\
Vert. beta function $\beta_{1,2}^{y}(\mathrm{~m})$ & 30.9 & 110.1 \\
Round $e$-beam radius $a_{1,2}(\mathrm{~mm})$ & 1.2 & 1.2 \\
Length of the $e$ beam $L(\mathrm{~m})$ & 2 & 2 \\
\hline \hline
\end{tabular}




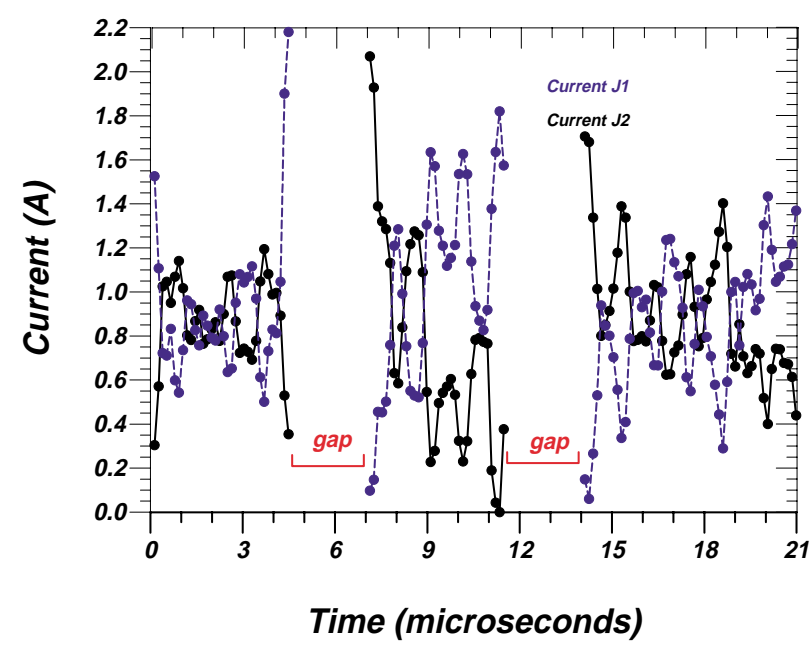

FIG. 4. (Color) Currents in the two electron lenses to compensate the bunch-to-bunch tune spread in the $140 \times 121$ bunches scenario; see text.

\section{B. Compensation of nonlinear beam-beam effects}

The interaction with other than a wide constant-density electron beam will not only shift the antiproton beam tunes, but will also distort the $\bar{p}$ footprint in a way that depends on the transverse electron charge distribution, $e-\bar{p}$ separation, crossing angle in the setup, etc. Let us consider the simplest example of the footprint due to "head-on" collisions of round Gaussian equal size proton and antiproton beams as shown in Fig. 6 . There, the large leaf shows betatron horizontal and vertical tunes (in units of the beam-beam parameter $\xi^{p} \equiv$ $\left.N_{\text {IP }} N_{p} r_{p} / 4 \pi \varepsilon_{n}\right)$ for antiprotons with different betatron oscillation amplitudes. For example, a zero amplitude

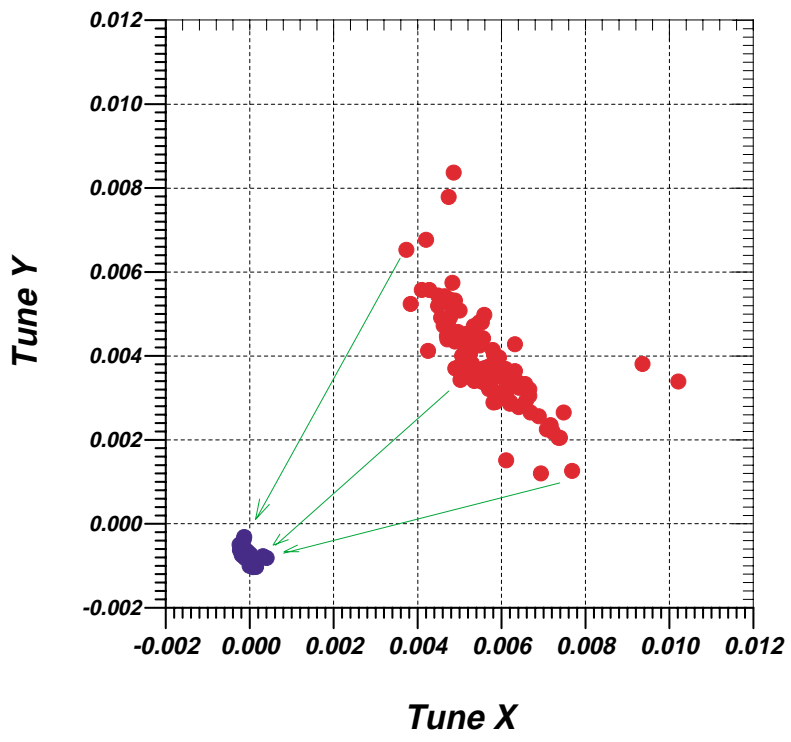

FIG. 5. (Color) Resulting $\bar{p}$ bunch tune shifts (core particles only) with $10 \%$ error of the compensation. particle has tunes $\Delta \nu_{z}(0,0)=+\xi^{p}$, very large amplitude particle tunes are not shifted at all $\Delta \nu_{z}(\infty, \infty)=0$, and tunes for horizontal and vertical amplitudes of $(1,2,3,4) \times$ the rms beam size $\sigma$ are presented and labeled.

The spread of the betatron frequencies (footprint) may enhance dynamic diffusion of particles due to high order resonances, increase radiation background in detectors, and limit beam lifetime and luminosity.

With the use of an electron beam one can, in principle, shrink the $\bar{p}$ footprint to a point if (a) the electron transverse charge distribution $\rho_{e}(r)$ is the same as in the proton beam $\rho_{p}(r)$, (b) the $\bar{p}$ beam distribution at the electron compressor is the same as at the IPs (but scaled in size and with zero dispersion), and (c) the total electron beam charge $e N_{e}=J L /\left(\beta_{e} c\right)$ on the path of the $\bar{p}$ beam (e.g., over the length $L$ of the central solenoid in Fig. 2) satisfies the equality condition of the beam-beam tune shifts. This equality condition for protons and electrons is

$$
\xi^{e} \equiv-\frac{N_{e} r_{p}\left(1+\beta_{e}\right)}{4 \pi \varepsilon_{n}}=-\xi^{p} .
$$

For simplicity, if we assume equal horizontal and vertical emittances and beta functions for antiprotons at the electron beam "compressor" device, then from Eq. (5) we get

$$
N_{e}=N_{\mathrm{IP}} N_{p} /\left(1+\beta_{e}\right) .
$$

This gives for TEV33 $N_{e} \approx 4.5 \times 10^{11}$ for $\beta_{e}=0.2$. For $L=3 \mathrm{~m}$ one needs $J=1.44 \mathrm{~A}$.

Evidently, it would be ideal to collide all three beams in one interaction point. Then, under conditions (a), (b),

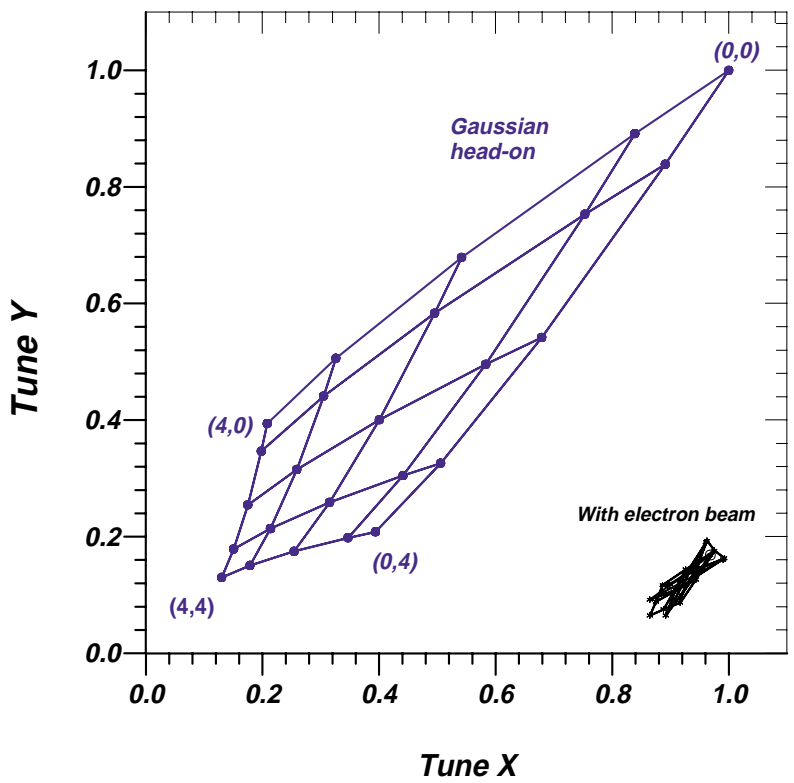

FIG. 6. (Color) Electron compression of the head-on $\bar{p}$ footprint. Tunes are given in units of the head-on beam-beam parameter $\xi^{p}$. Numbers in parentheses show the horizontal and vertical betatron amplitudes in units of the rms antiproton beam size. The case with electron beam is displaced for clarity. 
and (c) the electromagnetic force due to protons can be compensated by electrons and the antiprotons would experience no total kick at the IP. Unfortunately, in that case the nonrelativistic electron beam will affect protons almost as effectively as antiprotons (see Sec. IV D) and it is not desirable. In addition, stability of the electron and antiproton beams (see Secs. III and IV) prefers larger electron and antiproton beam sizes and lower current densities. Since the size is about the proton beam size, the electron beam is better installed at a large beta function location, e.g., at $\beta_{z}=200$ where $\sigma_{z} \approx 0.8 \mathrm{~mm} \mathrm{rms}$, and it definitely should not be set at the interaction point, where the sizes are the smallest (about $30 \mu \mathrm{m} \mathrm{rms}$ at the IP $\beta^{*}=$ $37 \mathrm{~cm}$ ) and vary over distances of about the bunch length $\beta^{*} \sim \sigma_{s}$. Moreover, there is no space at the interaction region for the necessary additional equipment. Good candidates are some locations near the Tevatron IPs where the beta functions can be as big as $\beta_{p} \simeq 1000 \mathrm{~m}$, but at present there is no available space in the superconducting magnet lattice for the electron beam setup at the locations where the horizontal and vertical $\beta_{p}$ are the same, and the dispersion function is equal to zero. Zero dispersion is desirable to avoid the possibility of synchrobetatron effects. Another possibility is to set the device at some other location, most probably in one of the Tevatron straight sections. The ideal straight section would provide (a) equal horizontal and vertical beta functions, (b) zero (or minimum) dispersion over the region of interaction with the electron beam, and (c) betatron phase advances between the IP and the electron beam setup to be multiple of $2 \pi$.

Rather effective footprint compression can be achieved even with non-Gaussian electron charge distributions. For example, Fig. 6 demonstrates the beam footprint compressed by an electron beam with charge density profile proportional to $\rho_{e}(r) \propto \frac{0.83}{1+(r / \sigma)^{8}}$ as shown by the line marked by crosses in Fig. 7. For convenience of presentation we have separated the smaller footprint horizontally (in fact it would be around zero tune point $\nu_{(x, y)}=0$ ) from the larger "leaf" due to head-on collisions with round Gaussian proton beam with charge distribution $\rho_{p}(r)=$ $\left(e N_{p} / 2 \pi \sigma^{2}\right) \exp \left(-\frac{r^{2}}{2 \sigma^{2}}\right)$ - as shown by the solid line in Fig. 7. One can see a significant reduction (6 times) of the tune spread with use of the electron beam.

Of course, the picture of the footprint compression is idealized. The beam-beam footprint itself can be significantly distorted by "imperfections" such as a crossing angle at the interaction point, or numerous parasitic interactions in multibunch colliders at the locations where two beams do not actually collide but still interact via long-range electromagnetic forces. The collider focusing lattice itself is not linear and this should also be taken into account. An additional difficulty is that nonlinearities are numerous and distributed over the collider ring while only one or very few electron beam setups can be installed.

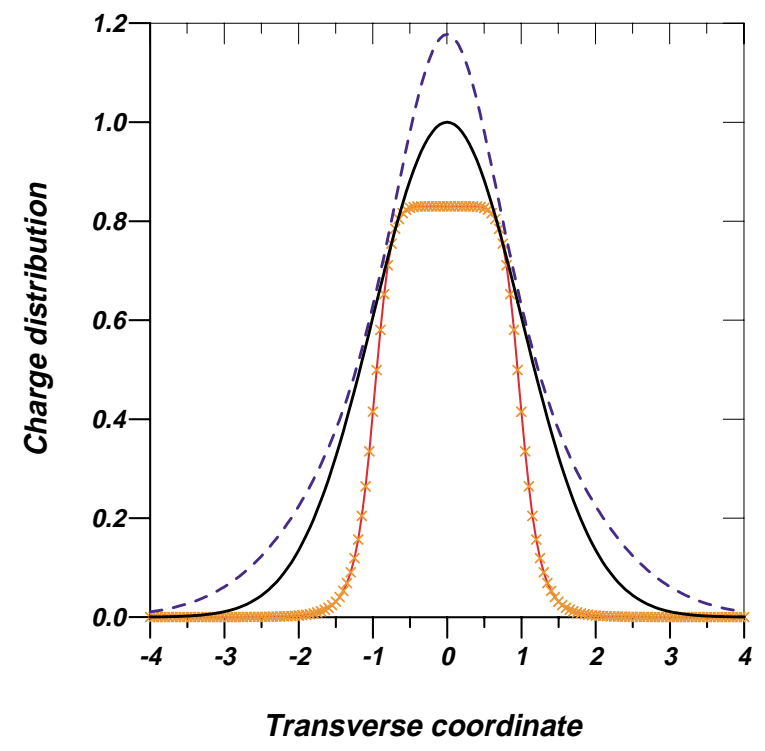

FIG. 7. (Color) Charge distributions. The solid line is Gaussian for antiprotons; $X$-marked line is the electron beam with $\rho_{e}(r) \propto \frac{0.83}{1+(r / \sigma)^{8}} ;$ dashed line is optimized electron beam distribution; see Eq. (8) and text.

Finally, it does matter what the ratio of the electron beam length is to the beta function at its location. Indeed, the proton bunch length of about $\sigma_{s} \simeq 37 \mathrm{~cm}$ is comparable to beta function at the interaction point $\beta^{*}$. Therefore, the betatron phase advance for antiprotons at the main IP is large $\psi_{z}=\int d s / \beta_{z} \simeq \sigma_{s} / \beta^{*} \simeq 1$. In contrast, the electron beam length of about $2-3 \mathrm{~m}$ is much less than the beta function at the compensation setup, and the corresponding betatron phase advance of antiprotons passing the electron beam is very small $\psi_{z} \simeq 0.01-0.02$. Thus, the electron beam kick looks like a delta function when transformed to the main IP. Consequently, such a short impact from the electrons contains a lot of resonance harmonics, although the average actions due to proton and electron beams are the same. One can reduce the betatron tune spread with a nonlinear lens, but this alone does not assure that the motion is more stable than that with no compensation, because the resonance strengths sometimes can be more important than the tune spread.

The electron beam setup can use several "knobs" in order to obtain better compensation: the transverse distribution $\rho_{e}(r)$, the separation of the electron beam from the $\bar{p}$ orbit, the angular separation between the beams, and the choice of the horizontal/vertical antiproton beta functions in the electron beam region. There are also a few less flexible options such as variation of the electron beam radius $a$ along the setup and the installation of several electron beam devices. For any specific goal (e.g., head-on beam-beam interaction compensation) only a few of the knobs need to be implemented for the device to be useful. 
In Ref. [9] we discuss the possibility of adding a single thin nonlinear lens to some arbitrary nonlinear lattice in such a way that the particle motion in the modified structure would become resonance free, though nonlinear, and the beam of particles would have a zero footprint. A numerical method to construct such maps is as follows: let us choose the tune equal to a resonant one, e.g., the 20 th order resonance. There is a simple way to determine whether the motion of a particle with each particular initial condition has that frequency or not: one calculates the squared differences of coordinates and momenta at the beginning and at the end of 20 successive map transformations. For example, in the normalized variables $x=\sqrt{\varepsilon} \cos (\psi)$ and $x^{\prime}=-\sqrt{\varepsilon} \sin (\psi)(\psi$ is the betatron phase), one takes the summation over some region of initial conditions and gets an optimization function $\tilde{F}$

$$
\tilde{F}=\sum_{j} \frac{\left(x_{f}-x_{i}\right)^{2}+\left(x_{f}^{\prime}-x_{i}^{\prime}\right)^{2}}{x_{i}^{2}+\left(x_{i}^{\prime}\right)^{2}},
$$

where symbols $i$ and $f$ are for initial and final normalized coordinates and angles, respectively, and the index $j$ denotes different phase space elements of initial conditions. The denominator of this expression is added in order to give trajectories with small and large amplitudes the same weight. When this function is equal to zero $\tilde{F}=0$, (i) the frequencies of all particles are equal to the particularly chosen value (1/20 in our case), and (ii) the strength of the resonance is equal to zero.

For resonant islands we get the same average frequency for all phase space elements of the island, but the motion inside the island has its own frequency. It gives a nonzero difference of initial and final conditions after the number of turns is equal to the number of the resonance (20 turns in this case). When the function $\tilde{F}$ is equal to zero, the motion inside the island is degenerate, so the resonance strength is equal to zero.

A numerical code has been developed that deals with minimization of the function $\tilde{F}$ by variation of the transverse electron charge distribution. This is chosen as a sum of Gaussian distributions with different rms values

$$
\rho_{e}(r)=\sum_{n=1}^{6} C_{n} \exp \left(-\frac{n r^{2}}{4 \sigma^{2}}\right),
$$

here $C_{n}$ are variable coefficients for optimization and $\sigma$ is the rms transverse proton beam size at the location of the electron beam. In simulations the electron beam produces a delta-function kick because of its short effective length, while the proton bunch length is presented as a number of short slices. The numerical code finds coefficients $C_{n}$ depending on the proton bunch length and the beam-beam parameter $\xi^{p}$. The synchrotron amplitude of antiprotons is taken to be zero. In the process of optimization the value $\tilde{F}$ usually decreases by a factor of 1000 .

For example, for $\sigma_{s}=2 \beta^{*}$, equal horizontal and vertical beta functions $\beta_{x}^{*}=\beta_{y}^{*}=35 \mathrm{~cm}$, tune shift (due to protons) $\xi^{p}=0.05$, the optimization results in coefficients $C_{1}=0.576, C_{2}=0.048, C_{3}=0.08, C_{4}=$ $0.042, C_{5}=0.04, C_{6}=0.4$ in the units of a Gaussian proton charge distribution corresponding to $C_{2}=1, C_{1}=$ $C_{3}=C_{4}=C$ (etc.) $=0$. The dashed line in Fig. 7 displays the resulting "optimized" distribution, and the solid line is for the Gaussian distribution.

\section{Electron compressor to eliminate crossing angle}

In principle, the "electron compression" allows for the elimination of the crossing angle at the interaction point. At present, a crossing angle is believed to be the best way to reduce the beam-beam tune shift and tune spread due to near-IP interactions. (Parasitic collision spacing is about $20 \mathrm{~m}=\frac{1}{2} c \times 132 \mathrm{~ns}$ ). A full crossing angle at the IPs of about $0.2 \mathrm{mrad}$ reduces the luminosity by about $50 \%$ [2].

Two or four near-IP head-on collision points will result in about 3 or 5 times larger tune shift and tune spread. Therefore, a proportional increase of the electron beam current $J$ from about 1.5 to $4.5-7.5$ A would be required to compensate these additional crossings. The only limitation is that in order to keep the higher current beam stable, a stronger magnetic field is needed. As shown in Sec. IV, the necessary field is approximately proportional to the tune shift due to electrons and, thus, to the current.

By eliminating the need for a crossing angle, this highcurrent electron compression can recover a factor of 2 in the luminosity, or provide the same luminosity with half the antiprotons. Two or four additional interaction points at high beta functions cost little in the rate of pbar consumption since the luminosity at these two additional head-on collision points is small $\mathcal{L} \propto 1 / \beta, \beta \gg \beta^{*}$.

\section{ELECTRON BEAM FOR BEAM-BEAM COMPENSATION}

As mentioned above, our beam-beam compensation proposal is based on a low energy (dozen kV), highcurrent (few A) electron beam colliding with antiprotons in a strong magnetic field of several Tesla; see Fig. 2. The electron source is the oxide cathode of an electron gun immersed in a comparatively weak magnetic field. The electrons enter a strong solenoid magnetic field straight interaction region experiencing adiabatic beam size compression which allows an increase in the current density. At the setup exit, the beam follows the magnetic field lines directed to the collector. In general, such a configuration is similar to "electron cooling" devices [10]. In this section we consider the electron beam itself, and start with the choice of electron energy.

The electron energy $U_{e}=m \beta_{e}^{2} c^{2} / 2$, or, equivalently, the electron velocity $\beta_{e}$, is a trade-off among several factors. Some of them, such as space-charge potential, drift ion instability, current modulation time, etc., prefer higher $U_{e}$ and $\beta_{e}$; others, e.g., power of the electron gun 
and the modulator power supply, adiabatic compression, and total beam current, prefer smaller beam energy.

Let us start with the general requirements on the electron beam for beam-beam compensation. First, its size must be about the same or few times the rms $\bar{p}$ beam size $\sigma_{z}=\sqrt{\beta_{z} \varepsilon_{n \bar{p}} / \gamma_{\bar{p}}}$. For the small emittance of TEV33 $\varepsilon_{n \bar{p}}=2.5 \times 10^{-6} \mathrm{~m}$ one gets $\sigma_{z}=0.51 \mathrm{~mm}$ at a beta function $\beta_{z} \simeq 110 \mathrm{~m}$ (see Table II). If one modifies the existing lattice and provides a high-beta, zero-dispersion region in the Tevatron, one would have $\sigma_{z}=0.77 \mathrm{~mm}$ at $\beta_{z}=250 \mathrm{~m}$. For simplicity, we will consider an electron beam with radius of $a=1 \mathrm{~mm}$ and constant transverse distribution. From Eqs. (3) and (6), the electron beam current $J$ scales with electron velocity as

$$
J=J_{0} \frac{\beta_{e}}{1+\beta_{e}},
$$

where the constant $J_{0} \approx 9.9$ A for the $2 \mathrm{~m}$ long electron lens discussed in Sec. II. The maximum current of a spacecharge limited diode electron gun is given by the ChildLangmuir law (see, e.g., [11])

$$
J=\mathcal{P} U_{a}^{3 / 2},
$$

where the perveance $\mathcal{P}$ is a gun geometry dependent parameter, and $U_{a}$ is the voltage difference between the cathode and the anode electrodes of the gun. Usually, perveance is presented in units of microperveance $\mathcal{P}=$ $\mu \mathcal{P} \times 10^{-6} \mathrm{~A} / \mathrm{V}^{3 / 2}$. In our case $U_{e} \simeq U_{a}$ and combining Eqs. (9) and (10) we get a minimum electron energy of

$$
U_{e} \approx \frac{1.2 J_{0}}{\mathcal{P} \sqrt{m c^{2}}}=\frac{16.3[\mathrm{kV}]}{\mu \mathcal{P}} .
$$

The electron lens requires modulation of the electron current with a characteristic time of $\tau \simeq 132 \mathrm{~ns}$. This can be done by varying the cathode-anode voltage $U_{a}$ from zero to $U_{e}$. If the cathode anode capacitance is approximately $C_{a}=20 \mathrm{pF}$, then the reactive power in the modulator circuit is about $W_{m}=C_{a} U_{e}^{2} /(2 \tau) \approx$ $20[\mathrm{~kW}] / \mu \mathcal{P}^{2}$. Thus, a higher gun perveance is beneficial for beam current modulation. The corresponding beam current and power are

$$
J \approx \frac{2.1[\mathrm{~A}]}{\sqrt{\mu \mathcal{P}}}, \quad W=J U_{e}=\frac{34[\mathrm{~kW}]}{\mu \mathcal{P}^{3 / 2}} .
$$

The energy recirculation technique used in electron cooling devices [10] allows for a reduction in the power dissipated on the collector. However, the high voltage power supply still has to provide power proportional to the total current $W_{c}=J U_{c}$, where $U_{c}=1 \ldots 2 \mathrm{kV}$ is the cathode-collector potential difference. Therefore, since it is beneficial to reduce the beam current and power, a high gun perveance is needed.

For a diode gun with a flat cathode and a Pierce electrode, the microperveance is equal to [11] $\mu \mathcal{P}=$ $7.3(a / d)^{2}$, where $a$ is the beam (cathode) radius and $d$ is the cathode-anode distance. A rule of thumb is that a good current density homogeneity can be reached if the ratio of $(a / d)$ is less than $1 / 2$, i.e., $\mu \mathcal{P}$ is less than 2 . Several times higher perveance (up to 10) with good beam quality can be achieved by the use of a convex cathode immersed in a magnetic field of the order of $1 \mathrm{kG}$ [12]an arrangement quite appropriate for our purposes. We rely on the possibility of making the electron gun for the electron lens with $\mu \mathcal{P}$ between 1 and 3, corresponding to $U_{e}$ from $16 \mathrm{kV}$ to $5.5 \mathrm{kV}$, while for numerical estimates elsewhere we use $\beta_{e}=0.2$ and $U_{e}=10 \mathrm{kV}$.

\section{A. Lower limit on the electron energy}

The lower limit on the electron energy is due to two effects. First, the electrons must be fast enough to provide the necessary current modulation, and, second, the electron kinetic energy must overcome the electron spacecharge potential in a round vacuum chamber.

Let us consider the time structure of the defocusing kick (or the tune shift) produced by the electron lens. Figure 8 demonstrates the effect of a steplike current modulation with pulse duration of $t_{p}$ (presented in the upper plot) on the antiproton bunches. Let us denote $t=0$ the moment when the front of the electron pulse enters the interaction section. As the antiproton beam passes through the oncoming electron current pulse, the maximum deflection will be seen by test particles which at $t=0$ are distanced by $\left(1+\beta_{e}\right) L / \beta_{e}$ from the input end of the device. We define the corresponding time $\tau_{g}=\left(1+\beta_{e}\right) L / c \beta_{e}$ as the "kick growth time." The maximum kick lasts over a time interval of $t_{f}=t_{p}-\tau_{g}$ which is synchronized with the bunch arrival (see lower diagram in Fig. 8). Behind that bunch, the kick amplitude vanishes over the growth time. The analytical expression

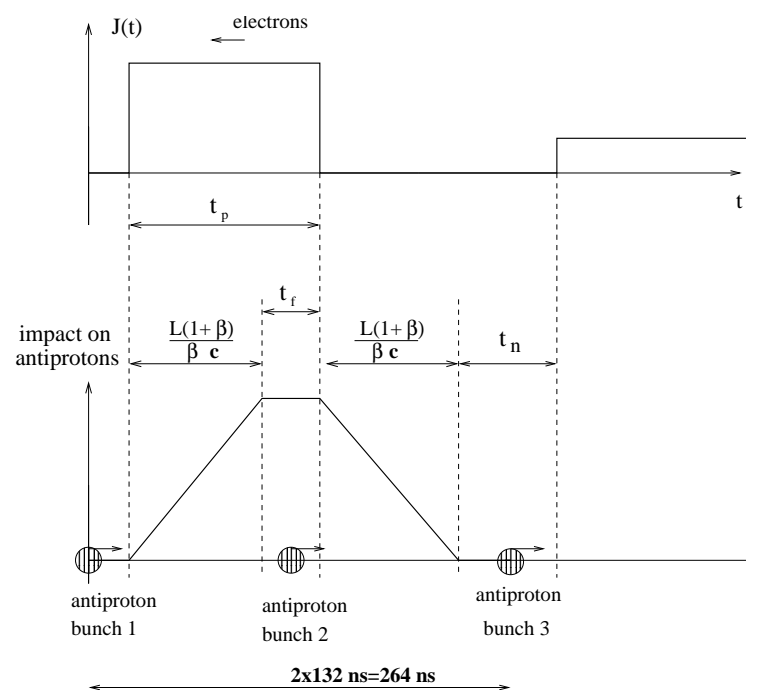

FIG. 8. The electron current modulation scheme. 
for the tune shift is as follows:

$$
\xi^{e}(t) \propto \int_{-t+2 \max \left[0, t-\left(1+\beta_{e}\right) l / c \beta_{e}\right]}^{t} J\left(t^{\prime}\right) d t^{\prime} .
$$

Let the required flattop of the kick acquired by $\bar{p}$ s be $t_{f}=5 \mathrm{~ns}$, and the required "no-impact time" to be the same $t_{n}=5 \mathrm{~ns}$. Then, summarizing all times in Fig. 8, the condition of $264 \mathrm{~ns}>t_{f}+2 L\left(1+\beta_{e}\right) / c \beta_{e}+t_{n}$ must be satisfied in order to have no impact on preceding and following bunches. This gives $\beta_{e}>0.06$ for $L=$ $2 \mathrm{~m}$ or corresponding to a kinetic energy $U_{e}>0.9 \mathrm{kV}$. Since one needs to modulate the electron current in order to equalize the bunch-by-bunch tune shift, the electrons have to be fast enough to provide different defocusing kicks on different bunches. $\beta_{e}=0.2$ satisfies the requirement.

One can make two remarks: first, if the current pulse duration is less than the growth time $t_{p}<\tau_{g}$, then the electron beam does not work in full strength; second, for a bunch spacing in the ring of $\tau$, the electron current pulse duration must be less than $t_{p}<2 \tau-2\left(1+\beta_{e}\right) L / c \beta_{e}$, otherwise, neighboring bunches will be affected. Thus, a rectangular pulse duration of $t_{p}=\tau_{g}$ corresponds to the device maximum strength. The length of the electron beam has to be less than $L<c \beta_{e} \tau / 2\left(1+\beta_{e}\right)$ because the current pulse shape cannot be exactly rectangular, and some flattop of the kick is required.

An example for TEV33 yields $\tau=132 \mathrm{~ns}, \beta_{e}=0.2$, and $L=2 \mathrm{~m}$. This satisfies the condition of $\tau_{g}=$ $40 \mathrm{~ns}<\tau$ and the requirement on the pulse length is $t_{p} \leq 264-40=224 \mathrm{~ns}$. Since the pulse shape of the current modulation cannot be exactly rectangular, the pulse full width at half amplitude has to be somewhat smaller (but still longer than $\tau_{g}$ ), e.g., $120-180 \mathrm{~ns}$.

The other limit on the minimum voltage (kinetic energy) is set by the electron beam space-charge potential $U_{\mathrm{sc}}$ with respect to the grounded vacuum chamber walls

$U_{\mathrm{sc}}=\frac{2 e N_{e}}{L}\left[\ln \left(\frac{b}{a}\right)+\frac{1}{2}\right] \approx 500[\mathrm{~V}]\left[\ln \left(\frac{b}{a}\right)+\frac{1}{2}\right]$,

where $N_{e}=J L /\left(e \beta_{e} c\right) \approx J_{0} L /(1.2 e c)$. Accordingly to Ref. [13], the stability of the electron beam with size much less than the vacuum pipe radius requires $U_{e}>$ $\frac{3}{2} U_{\mathrm{sc}}=3.1 \mathrm{kV}$ for a chamber radius of $b=40 \mathrm{~mm}$ and $a=1 \mathrm{~mm}$ beam radius.

\section{B. Electron beam in a magnetic field}

The electron lens setup needs to have a longitudinal magnetic field in order to keep the electron beam envelope stable, make the beam more rigid, transport it from the cathode through the interaction region to the collector, and obtain a smaller beam size. The equation for paraxial electron oscillations under the impact of a solenoidal field $B$, the space-charge force due to the electron beam, and the force due to incoming antiprotons is

$$
\frac{d^{2} r}{d s^{2}}+r\left(\frac{1}{F_{B}^{2}}-\frac{1}{F_{e}^{2}}-\frac{1}{F_{\bar{p}}^{2}}\right)=0,
$$

where $s$ is the longitudinal coordinate along the electron orbit, and $r=|x+i y|$ is the oscillation amplitude (see Refs. $[14,15]$ for a detailed analysis of electron flows). The effective focal length due to the magnetic field $B$ is

$$
F_{B}=\frac{2 \gamma_{e} \beta_{e} m_{e} c^{2}}{e B} \approx 3.3[\mathrm{~cm}] \frac{\gamma_{e} \beta_{e}}{B[\mathrm{kG}]},
$$

which for $10 \mathrm{kV}$ electrons yields $F_{B}=$ $0.66[\mathrm{~cm}] / B[\mathrm{kG}]$. It has to be compared with the defocusing length due to electron space charge which is given by

$F_{e}=\sqrt{\frac{m c^{3} \gamma_{e}^{3} \beta_{e}^{3} a_{e}^{2}}{2 e J}} \approx 2.9[\mathrm{~cm}] \beta_{e} \gamma_{e}^{3 / 2} \sqrt{1+\beta_{e}}$,

where we use Eq. (9) for the required electron current. The minimum defocusing length due to the antiproton beam is

$$
F_{\bar{p}}=\sqrt{\frac{\gamma_{e} \beta_{e}^{2} \sqrt{2 \pi} \sigma_{s} \sigma_{\bar{p}}^{2} m c^{2}}{e^{2} N_{\bar{p}}\left(1+\beta_{e}\right)}} \approx 4.1[\mathrm{~cm}] \beta_{e} \sqrt{\frac{\gamma_{e}}{1+\beta_{e}}},
$$

where we take $N_{\bar{p}}=6 \times 10^{10}, \sigma_{\bar{p}}=0.9 \mathrm{~mm}$ (the $\bar{p}$ beam size), and $\sigma_{s}=14 \mathrm{~cm}$ (the rms bunch length). For $10 \mathrm{kV}$ electrons $F_{B}=0.66[\mathrm{~cm}] / B[\mathrm{kG}], F_{e}=0.64 \mathrm{~cm}$, and $F_{\bar{p}}=0.74 \mathrm{~cm}$. The beam envelope oscillations are stable if the focusing term in Eq. (15) is stronger than the two defocusing terms

$$
\frac{1}{F_{B}^{2}} \geq \frac{1}{F_{e}^{2}}+\frac{1}{F_{\bar{p}}^{2}}
$$

The required magnetic field for a $10 \mathrm{kV}$ electron beam is $1.4 \mathrm{kG}$. Since the device uses the electron beam once over a passage, then, in principle, one could consider using no magnetic field at all if the electron beam energy is high enough to have only minor electron beam disruption over the length of the pbar bunch $F_{\bar{p}} \gg \sigma_{s}$. For $\sigma_{s}=14 \mathrm{~cm}$ this yields an electron kinetic energy of $U_{e} \gg 12 \mathrm{MeV}$. Taking into account the high average current, one can see that the electron beam power exceeds dozens of MW. This makes the use of relativistic electron beams very impractical for the beam-beam compensation.

As we will discuss later in Sec. IV, a much stronger solenoid field of the order of 4-6 T is necessary to maintain stability of the antiproton beam and reduce $x-y$ coupling due to electron beam distortions. In such a strong field, magnetic focusing dominates the electron dynamics. Each electron performs very fast Larmor oscillations with frequency $\omega_{L}=e B /\left(\gamma_{e} m c\right)$ and spatial period of $\lambda_{L}=2 \pi v_{e} / \omega_{L}=\pi F_{B} \approx 2.1[\mathrm{~cm}] / B[\mathrm{kG}] \simeq 0.5 \mathrm{~mm}$. Their orbits can be represented as tiny (micron scale) Larmor circles moving along the magnetic field line. 
The effect of a space-charge field $\vec{E}$ is that each of these circles starts to rotate slowly (drift) around the beam axis while staying at the same radius, i.e., the round beam remains round. The drift velocity in crossed electric and magnetic fields $\vec{E}$ and $\vec{B}$ is equal to

$$
\vec{v}_{d}=c \frac{[\vec{E} \times \vec{B}]}{B^{2}} .
$$

The space-charge electric field inside a constant current density electron beam with $j_{e}=J / \pi a^{2}$ is proportional to radius $\vec{E}=2 j_{e} \vec{r} / \beta_{e}$. The angle $\theta_{d}$ of the drift rotation over the time interval $t$ does not depend on radius $\theta_{d}=v_{d} t / r=2 j c t / \beta_{e} B$. For example, the angle over the beam passage of $L=2 \mathrm{~m}$ in a $B=40 \mathrm{kG}$ field is about $\theta_{d} \approx 240^{\circ}$. The electric field due to a Gaussian $\bar{p}$ beam is not linear, and the rotation angle $\theta_{d}$ is no longer independent of $r$, and electrons with larger $r$ perform drift rotation on different (smaller) angles. However, the difference is negligible for our parameters - see detailed studies in Ref. [16]. The magnetic forces due to electron and antiproton currents produce additional drifts similar to electric ones, but their contributions are $\beta_{e}^{2}$ and $\beta_{e}$ times smaller, respectively, and, therefore, negligible.

The required current density for a $2 \mathrm{~mm}$ diameter beam is $j_{e}=\frac{J}{\pi a_{e}^{2}} \approx 315 \frac{\beta_{e}}{1+\beta_{e}} \mathrm{~A} / \mathrm{cm}^{2}$, or about $53 \mathrm{~A} / \mathrm{cm}^{2}$ for a $10 \mathrm{kV}$ electron beam. On the other hand, the oxide cathode lifetime goes down greatly if the current density exceeds $5-10 \mathrm{~A} / \mathrm{cm}^{2}$ (see Ref. [17] and references therein). To reduce the cathode current density limit, one can use adiabatic magnetic compression in which the beam is born on the cathode with a larger radius $a_{c}$ in a weak field $B_{c}$ and transported to the region of stronger magnetic field $B$, with conservation of the adiabatic invariant $B_{c} a_{c}^{2}=B a^{2}$. For the electron lens with cathode current density of about $2.1 \mathrm{~A} / \mathrm{cm}^{2}$ and $a_{c}=5 \mathrm{~mm}$, the maximum "shrinking" ratio $\mathcal{R} \equiv B / B_{c} \equiv a_{c}^{2} / a^{2}$ should be about 25 , e.g., $B=40 \mathrm{kG}, B_{c}=1.6 \mathrm{kG}$. This looks quite feasible technically.

Nonlinear beam-beam compensation and the footprint compression require precise control of the transverse electron charge distribution $\rho_{e}(r)$. A natural possibility is to do that using near cathode electrodes in the diode electron gun. If one applies a potential to these electrodes which is different from the cathode potential, then the distorted electric field distribution on the cathode surface will decrease (or increase) the electron emission from different radial areas of the cathode.

\section{Effect of ions}

While passing through the vacuum chamber, the electron beam ionizes residual gas atoms and produces electrons and positively charged ions. Under certain conditions both these electrons and ions may concentrate in the electron beam. This could result in (a) changing the total charge density within the beam, i.e., changing the effectiveness of the beam-beam compensation, and (b) developing the so-called two-beam drift instability. Measures should be applied to ensure proper removal of the residual particles.

Ionization of residual gas by electrons produces ions with the rate

$$
\frac{d n_{i}}{d t}=\sigma_{\mathrm{ioniz}} n_{e} v_{e} n_{0},
$$

where $\sigma_{\text {ioniz }}$ is the ionization cross section, $n_{e}$ is the electron beam density, and $n_{0}$ is the residual gas density. A useful quantity is the "neutralization time"

$$
\tau_{n}=\frac{1}{\sigma_{\mathrm{ioniz}} v_{e} n_{0}},
$$

the time in which the electron beam is fully neutralized if all produced ions remain in the beam. For $\beta_{e}=$ $0.2(10 \mathrm{kV})$ the total cross section (including initial, second, etc. ionization) for $N_{2}$ is approximately $\sigma_{\text {ioniz }} \approx$ $2 \times 10^{-17} \mathrm{~cm}^{2}$ [18]. At room temperature the residual gas density is $n_{0} \approx 3.2 \times 10^{16} \mathrm{P}$ (Torr) $\mathrm{cm}^{-3}$. So, the neutralization time is approximately

$$
\tau_{n} \approx 2.5 \times 10^{-10} / P \text { (Torr) } \mathrm{s} .
$$

For example, $\tau_{n} \approx 0.25 \mathrm{~s}$ if $P=10^{-9}$ Torr.

Drift instability is the main limitation on the beam current in the presence of ions. The origin of the phenomenon is the exponential amplification along the beam of a small initial separation of the electron and ion beams at certain frequencies. This amplification results in an instability if the amplification coefficient is larger than the feedback coefficient from the beam end to the beam beginning. Theoretical analysis of the instability is given in $[19,20]$ and agrees well with experimental investigations of the fully neutralized $\left(n_{i}=n_{e}\right)$ electron beam [19]. The stability threshold current density is found to be about

$$
j_{e}<\frac{v_{e}^{2} B}{3.8 L c} .
$$

Taking this expression one can estimate the acceptable ion density for the electron compressor

$$
\frac{n_{i}}{n_{e}}<\frac{v_{e}^{2} B}{4 L c j_{e}} \approx 0.8 \frac{B[\mathrm{kG}] \beta_{e}^{2} a^{2}[\mathrm{~mm}]}{L[\mathrm{~m}] J[\mathrm{~A}]} .
$$

If $\beta_{e}=0.2, B=40 \mathrm{kG}, a=1 \mathrm{~mm}, J=2 \mathrm{~A}, L=$ $2 \mathrm{~m}$, then $n_{i} / n_{e}<0.13$. Therefore, though the ions should be cleaned out from the beam in order to avoid instability, the value is rather relaxed.

A more stringent requirement follows from the condition that the total charge distribution remain controllable within at least a few percent, i.e., $n_{i} / n_{e}<0.01$.

The potential well of the electron beam prevents the residual ions from getting out of the beam in the transverse direction. The potential at the axis is given by $U_{\text {sc }}$ in Eq. (14). One can see that since the beam radius $a$ is smaller in the central part of the electron lens setup, then $U_{\mathrm{sc}}$ is bigger there than at the gun and collector, and this forms a longitudinal trap for ions. The ions 
can be removed from the beam if one ensures that either (a) ions may escape to the collector or gun, i.e., the ratio $b / a$ remains constant and the potentials at the collector or gun allow the ions to enter, or (b) one uses special sufficiently long cleaning electrodes to produce a transverse electric field $E_{c}$ larger than the field due to electron space charge $E_{c}>2 \pi a e n_{e}$ (see Fig. 9).

Let us now suppose that the residual ions can exit at the beam edges either via cleaning electrodes or the gun and collector, and let us assume that the ratio $b / a$ is constant along the beam. The ions are trapped and their density $n_{i}(s)$ increases until the influx of ions due to ionization is balanced by the loss due to their own longitudinal field $E=U_{e} / n_{e} d n_{i}(s) / d s$. The resulting approximate shape of the residual ion density looks like $n_{i}(s) \approx n_{i}^{\max }\left[1-(2 s / L)^{2}\right]^{1 / 3}$, with the maximum

$$
n_{i}^{\max }=n_{e}\left(\frac{4 \tau_{c} \omega_{i} a \sqrt{[1+2 \ln (b / a)] / 3}}{\pi L}\right)^{-2 / 3},
$$

where the ion plasma frequency is $\omega_{i}=\sqrt{2 \pi n_{i} e^{2} Z_{i} / M_{i}}$. Equations (22) and (26) give an estimation for the residual gas pressure if the limit on $n_{i} / n_{e}$ is set. For example, if $\beta_{e}=0.2, a=1 \mathrm{~mm}, b=30 \mathrm{~mm}, J=2 \mathrm{~A}, L=2 \mathrm{~m}$, and ions are single ionized nitrogen molecules, then the condition $n_{i}^{\max } / n_{e}<10^{-2}$ results in $\tau_{n}>0.028 \mathrm{~s}$, i.e., the vacuum should be better than $P<9 \times 10^{-9}$ Torr.

One should also mention several particular problems associated with the ionization electrons. These electrons are highly magnetized, so they can move only longitudinally and drift around the beam. Since both the electron gun and collector have negative potential with respect to the main vacuum chamber, the electrons are trapped. However, they have at least two ways to leave the beam. First, they are heated by the main beam until their energy increases enough to leave the potential well. The heating rate of this "electron wind" is $d\left(W / U_{e}\right) / d t \approx 4 L_{c} e^{2} / m v_{e} a^{2}[1+2 \ln (b / a)]$, where $L_{c} \sim 7$ is the Coulomb logarithm. Second, the center of

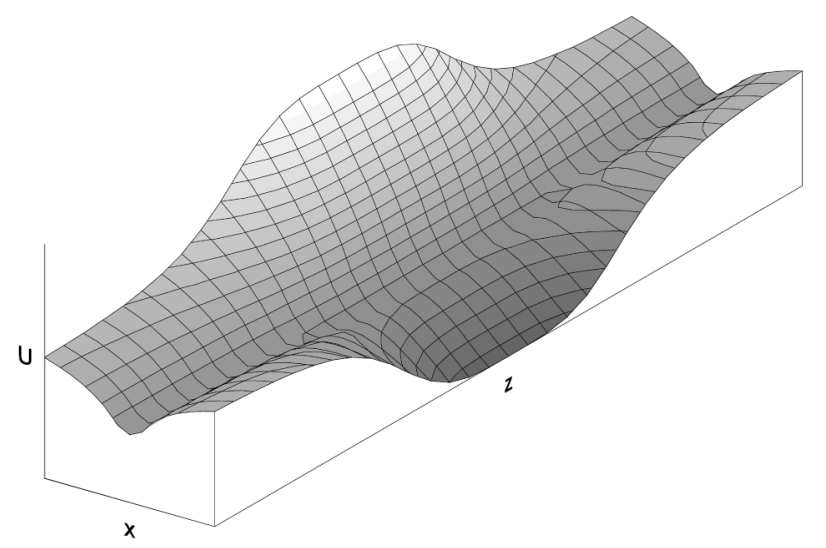

FIG. 9. Changing of the electron beam potential well by the cleaning electrode. the electron trajectory jumps by approximately a Larmor radius each time the electron is reflected from the potential wells of the electron gun or collector (if the Larmor circle step is larger than the length over which the potential changes). This results in a diffusive (or systematic if the axial symmetry is not perfect) loss of these electrons.

Another problem is that these ionization electrons may be trapped and stored in the vicinity of the cleaning electrodes and their charge may change significantly the distribution of potentials. It was found in [21] that this problem can be avoided if one uses insertions made from low conductive material such as a semiconductor glass (see Fig. 10).

\section{SIDE EFFECTS ON HIGH-ENERGY BEAMS}

\section{A. Electron beam distortions in beam-beam compensation setup}

As we have shown in the previous section, collision with a round antiproton bunch in a strong magnetic field conserves axial symmetry and the radial size of the electron beam. Therefore, the electron beam space-charge forces are the same for antiprotons at the head and at the tail of the antiproton bunch. This is no longer true if the electron or antiproton beam is not round. Axial symmetry of the electron beam can be assured by using a round cathode in the electron gun and by an appropriate choice of the magnetic field in the transport section of the setup. The antiproton beam roundness could be achieved in a number of Tevatron locations where vertical and horizontal beta functions are the same $\beta_{x}=\beta_{y}$. However, this condition cannot always be fulfilled; see, e.g., Table II for the proposed electron lens parameters.

The electron beam cross section becomes a rotated ellipse as the tail of a nonround antiproton bunch passes it, whereas the head of the bunch sees the original undisturbed round electron beam. Detailed numerical

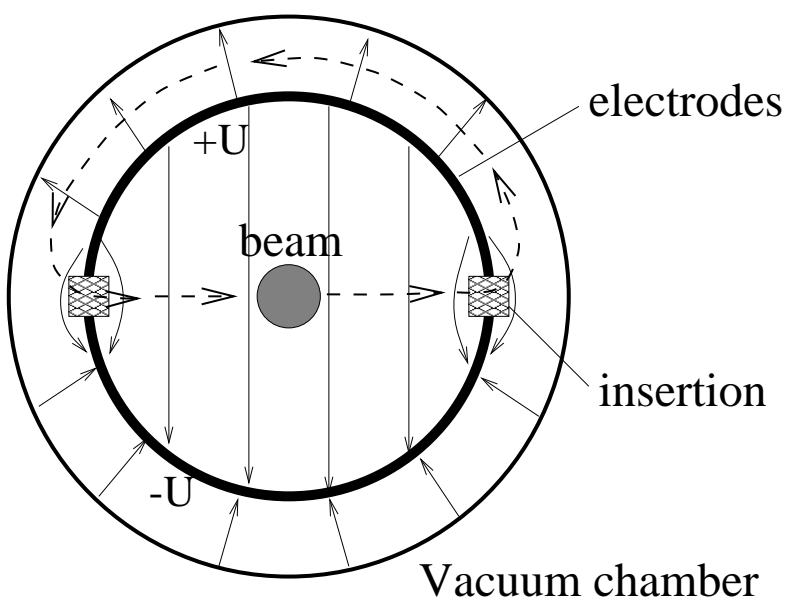

FIG. 10. Cleaning electrodes with low conductive insertions. Dashed lines show trajectories of trapped ionization electrons. 
studies of the effect can be found in Ref. [16]. The electron beam distortions are of concern because (a) the distortion of the space-charge forces which plays a role in the beam-beam compensation, (b) in addition to the desired defocusing effect, electric fields of the elliptic electron beam produce $x-y$ coupling of vertical and horizontal betatron oscillations in the antiproton beam, and (c) there appears a "head-tail" interaction in the antiproton bunch via higher order wake fields propagating in the electron beam.

The electron beam distortions can be found analytically. We start with the continuity equation for the electron charge density $\rho_{e}(x, y, s, t)$

$$
\frac{\partial \rho_{e}}{\partial t}+\operatorname{div}\left(\rho_{e} \vec{v}\right)=0
$$

where $\vec{v}(x, y, s, t)$ is the velocity of electrons. Since the longitudinal component of the velocity is constant $v_{s}=\beta_{e} c$ and all longitudinal scales (like the antiproton bunch length $\sigma_{s}$ or electron beam length) are much longer than the transverse scale, one can neglect the term $\partial / \partial s\left(\rho v_{s}\right)$ in (27). We have separated the fast small amplitude Larmor motion and the slow large amplitude drift with velocity $\vec{v}_{d}$ in Eq. (20). The latter is the major source of the electron beam distortion, and in the following analysis we consider $\vec{v}=\vec{v}_{d}$. If we assume the unperturbed electron charge distribution to be axially symmetric $\rho_{e}(t=0)=\rho_{0}(r)$, and that the maximum density distortion is small $\rho_{e}=\rho_{0}+\delta \rho, \delta \rho \ll \rho_{0}$, then in the lowest order one gets from (27)

$$
\frac{\partial \delta \rho}{\partial t}+\vec{v}_{d} \cdot \vec{\nabla} \rho_{0}+\rho_{e} \operatorname{div} \vec{v}_{d}=0
$$

The third term is equal to zero because $\operatorname{div} \overrightarrow{\boldsymbol{v}}_{d}=0$. The gradient in the second term can be written as $\vec{\nabla} \rho_{0}=$ $2 \vec{r} d \rho_{0}\left(r^{2}\right) / d\left(r^{2}\right)$, and thus, we obtain

$$
\overrightarrow{\boldsymbol{v}}_{d} \cdot \vec{\nabla} \rho_{0}=\frac{2 c}{B^{2}} \frac{d \rho_{0}\left(r^{2}\right)}{d\left(r^{2}\right)}[\vec{E} \times \vec{B}] \cdot \vec{r} .
$$

The electric field of the round electron beam does not contribute to the product above since it is proportional to $\vec{r}$. The contribution due to electron beam space charge can be ignored as long as the electron charge density distortions are small with respect to $\rho_{0}(r)$. The major reason for the density change $\delta \rho$ is the antiproton beam space-charge force. The electric field of the elliptic Gaussian relativistic antiproton beam with rms sizes $\sigma_{x}$ and $\sigma_{y}=R \sigma_{x}$ is given by

$$
\vec{E}=-e N_{\bar{p}} \lambda(s) \cdot \vec{\nabla} U,
$$

where the linear density of antiprotons is normalized as $\int \lambda(s) d s=1$, and the two-dimensional effective interaction potential $U(x, y)$ is [22]

$$
U(x, y)=\int_{0}^{\infty} d q \frac{1-\mathrm{e}^{-\frac{x^{2}}{2 \sigma_{x}^{2}(1+q R)}-\frac{y^{2}}{2 \sigma_{y}^{2}(1+q / R)}}}{\sqrt{(1+q R)(1+q / R)}} .
$$

Therefore, after some mathematics we get

$$
\delta \rho\left(x, y, t=\frac{s}{\left(1+\beta_{e}\right) c}\right)=\left(\int_{-\infty}^{s} \lambda\left(s^{\prime}\right) d s^{\prime}\right) \frac{2 e N_{\bar{p}}}{B} \frac{d \rho_{0}\left(r^{2}\right)}{d\left(r^{2}\right)} \frac{x y I(x, y)\left(\sigma_{x}^{2}-\sigma_{y}^{2}\right)}{\sigma_{x}^{2} \sigma_{y}^{2}}
$$

where now $s$ is the coordinate inside the antiproton bunch ${ }^{1}$ and

$$
I(x, y)=\int_{0}^{\infty} d q \frac{\mathrm{e}^{-\frac{x^{2}}{2 \sigma_{x}^{2}(1+q R)}-\frac{y^{2}}{2 \sigma_{y}^{2}(1+q / R)}}}{(1+q R)^{3 / 2}(1+q / R)^{3 / 2}},
$$

e.g., $I(0,0)=\frac{2 R}{(1+R)^{2}}$. The major features of the distortion are (a) it is absent in the case of a round antiproton beam when $\sigma_{x}=\sigma_{y}$, (b) it performs two variations over azimuth $\delta \rho \propto x y \sim \sin (2 \theta)$, (c) it diminishes as the solenoid field $B$ increases, or as the antiproton intensity $N_{\bar{p}}$ decreases, and (d) most of the distortion takes place at the radial edge of the electron beam, and, since $d \rho_{0}\left(r^{2}\right) / d\left(r^{2}\right) \simeq \rho_{0}^{\max } / a^{2}$, a wider electron beam receives smaller density distortions during the interaction;

\footnotetext{
$1_{\text {i.e., } s}=-\infty$ is for the bunch head and $\int_{-\infty}^{s} \lambda\left(s^{\prime}\right) d s^{\prime}$ is proportional to the antiproton charge which passed through the given part of the electron beam.
}

there is no distortion inside a round constant density electron beam. Finally, the scaling of the maximum distortion strength is

$$
\frac{\delta \rho^{\max }}{\rho_{0}^{\max }} \simeq \frac{0.2 e N_{\bar{p}}}{a^{2} B} \approx \frac{0.6\left[N_{\bar{p}} / 6 \times 10^{10}\right]}{a^{2}[\mathrm{~mm}] B[\mathrm{kG}]},
$$

where the value of 0.2 comes from the geometrical factor $\propto x y \cdot I(x, y)$. For example, the distortion is about $1.5 \%$ for a $1 \mathrm{~mm}$ radius electron beam in a $B=40 \mathrm{kG}$ solenoid field. Note that as soon as the elliptic distortion appears it starts a drift rotation in the crossed fields of the electron space charge and the solenoid field. It is important that during the passage of the antiproton bunch (about $\pm 2 \sigma_{s} / c=2 \mathrm{~ns}$ ), the rotation is small. For example, for $B=2 \mathrm{~T}$ the angle is about $\theta_{d} \approx 4 j \sigma_{z} a / \beta_{e} B \approx$ $0.1 \mathrm{rad} \ll 1$. Thus, ignoring the factor $\vec{v}_{d} \cdot \vec{\nabla} \delta \rho$ in Eq. (28) is justified.

Figure 11 shows the electron beam distortion calculated analytically with Eq. (32). The top left-hand plot in Fig. 11 shows contour lines of constant density for the 

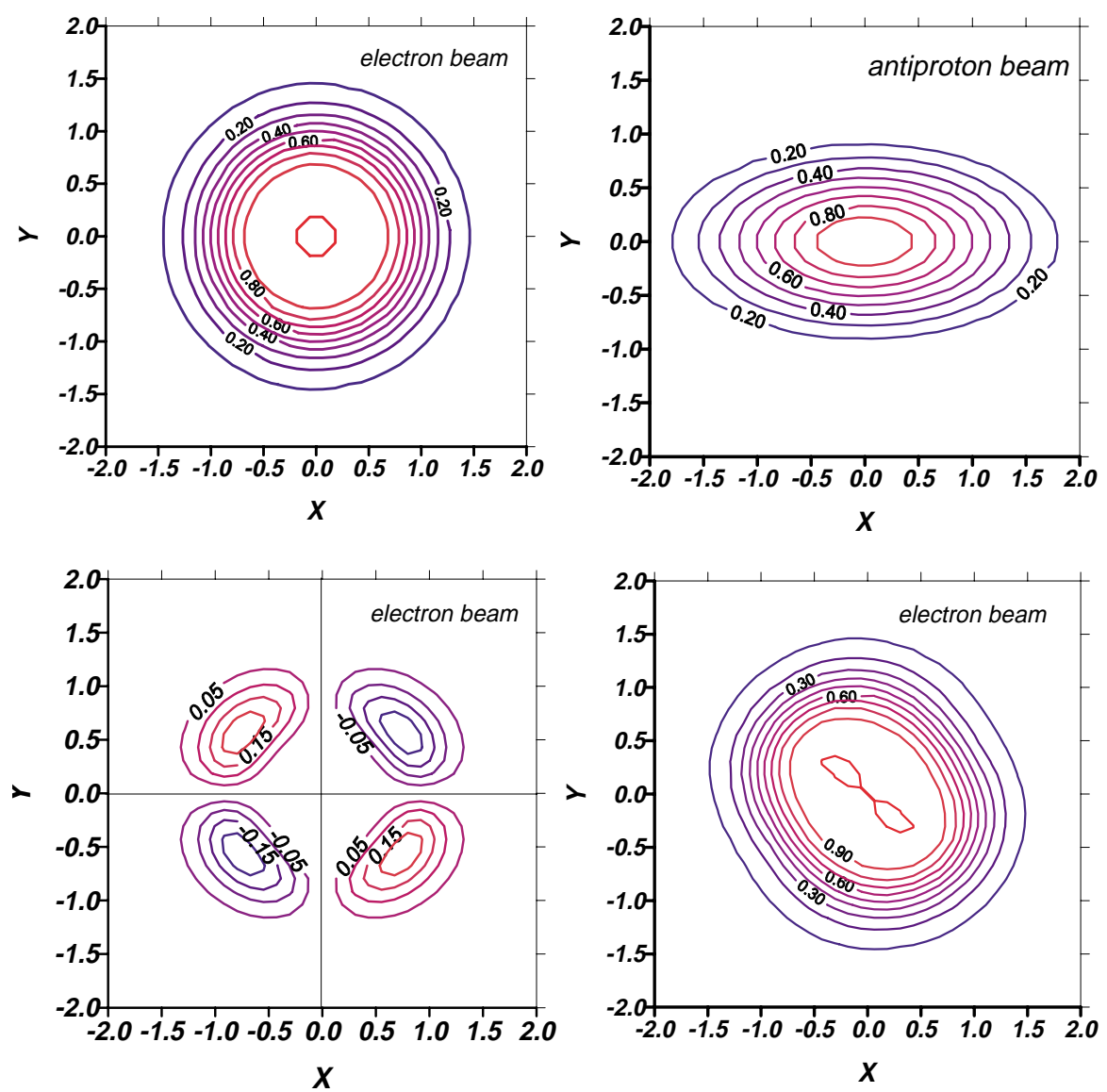

FIG. 11. (Color) Contour plots of original electron density (top left), antiproton density (top right), change of the electron density due to interaction with antiproton space charge (bottom left), and resulting electron density (bottom right). $x$ and $y$ coordinates are given in units of $\sigma_{x}$.

electron beam with a particular initial distribution of

$$
\begin{aligned}
& \rho_{0}(r)=\frac{1}{1+(r / a)^{2 \mu}}, \\
& \quad \mu=3, a=\sigma_{x}=0.61 \mathrm{~mm} .
\end{aligned}
$$

Here and below the $x$ and $y$ coordinates are given in units of $\sigma_{x}$

Constant density lines for the Gaussian distribution in the antiproton beam with $\sigma_{x}=0.61 \mathrm{~mm}$ and $\sigma_{y}=$ $0.31 \mathrm{~mm}$ are presented in the top right-hand plot. The lower left-hand corner of the figure shows the change of the electron charge density $\delta \rho(x, y)$ after passage through an antiproton bunch with $N_{\bar{p}}=6 \times 10^{10}$ in a magnetic field $B=4 \mathrm{kG}$. With such a small solenoid field the distortion is very large $\delta \rho^{\max } \simeq 0.25$ and the resulting electron beam shape $\rho=\rho_{0}+\delta \rho$ is clearly a rotated ellipse as shown in the lower right-hand plot. In this case, the space-charge fields are very different for the antiprotons in the head and in the tail of the bunch. The solenoid field in the electron lens setup will be about 10 times stronger $B \approx 40 \mathrm{kG}$, and, consequently, we expect 10 times smaller electron beam distortions.
As we noted in Sec. II, the electron beam has to be 2-3 times wider than the antiproton beam for adequate linearity in the electron lens setup. According to Eq. (34), this also helps to reduce $\delta \rho$ by a factor of $4-9$ [16].

\section{B. Coupling due to distorted electron beam}

Electric and magnetic fields of the elliptic electron beam lead to $x-y$ coupling of vertical and horizontal betatron oscillations in the antiproton beam. Since originally the electron beam is round, the head of the antiproton bunch experiences no coupling force. But, since the electron density distortion grows as $\int^{s} \lambda\left(s^{\prime}\right) d s^{\prime}$ [see Eq. (32)], the coupling grows proportionally. Particles in the head and in the tail of the bunch change their positions while performing synchrotron oscillations, thus, an average coupling effect is about half of the maximum coupling spread. The average coupling can be corrected in the Tevatron, but there are no available tools to compensate the spread in coupling. Therefore, the spread has to be small enough in order not to affect the antiproton beam dynamics. 
The tunes of a small amplitude particle can be written as

$$
\nu_{ \pm}=\frac{\left[\left(\nu_{x}+\Delta \nu_{x}\right)+\left(\nu_{y}+\Delta \nu_{y}\right)\right]}{2} \pm \sqrt{\frac{\left[\left(\nu_{x}+\Delta \nu_{x}\right)-\left(\nu_{y}+\Delta \nu_{y}\right)\right]^{2}}{4}+|\kappa+\Delta \kappa|^{2}},
$$

where $\nu_{x}$ and $\nu_{y}$ are the unperturbed horizontal and vertical tunes, $\kappa$ is a complex number describing the coupling, and $\Delta$ 's represent the changes of these quantities that arise from the interaction with the electron beam. The tunes in the current Tevatron lattice are $\nu_{x}=0.585$ and $\nu_{y}=0.575$. For satisfactory operation of the Tevatron collider, the global coupling is corrected down to a value of $|\kappa| \approx 0.001$ [23].

In terms of the two-dimensional potential $V(x, y)$, the horizontal tune shift is given by Eq. (1) - see Sec. IIand the coupling shift can be calculated as

$$
\Delta \kappa=\frac{\sqrt{\beta_{x} \beta_{y}} e^{i\left(\psi_{x}-\psi_{y}\right)}}{4 \pi} \frac{\partial^{2} V}{\partial x \partial y} .
$$

In the case of an almost round electron beam with a small elliptic distortion one can write $V(x, y)=V_{0}(r)+$ $V_{\text {skew }}(x y)$. The potential $V_{0}$ and corresponding tune shift for a round beam are given in Eqs. (2) and (3). Of course, a perfectly round electron beam gives no contribution to the coupling.

Now, let us write the electron density distortion in the form $\delta \rho(x, y)=x y \cdot C(x, y)$ which emphasizes the product $x y$ and the rest is a slowly varying function of $x y$,

$$
C(x, y)=\frac{2 e N_{\bar{p}}}{B} \frac{d \rho_{0}\left(r^{2}\right)}{d\left(r^{2}\right)} \frac{I(x, y)\left(\sigma_{x}^{2}-\sigma_{y}^{2}\right)}{\sigma_{x}^{2} \sigma_{y}^{2}} .
$$

The effective $2 \mathrm{D}$ skew potential can be found as a solution of following equation:

$$
\Delta V_{\text {skew }}=-4 \pi \delta \rho \frac{r_{\bar{p}}}{\gamma_{\bar{p}}},
$$

which is approximately equal to

$$
V_{\text {skew }} \approx \frac{\pi r_{\bar{p}}}{6 \gamma_{\bar{p}}} C(x, y) r^{2} x y .
$$

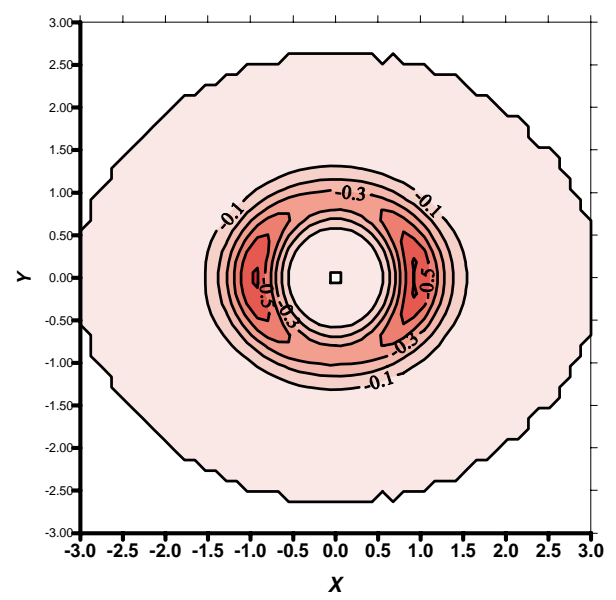

This yields a coupling magnitude of

$$
|\kappa| \approx \frac{\sqrt{\beta_{x} \beta_{y}}}{8 \gamma_{\bar{p}}}\left\langle C(x, y) r^{2}\right\rangle
$$

The brackets \langle\rangle denote averaging over antiproton betatron oscillations. One can estimate the maximum coupling spread using Eqs. (3), (37)-(41) together with the approximate relation $\beta_{x} \simeq 3 \beta_{y}$

$$
|\kappa| \approx \frac{\left|\xi_{e}\right| e N_{\bar{p}}}{2 \sqrt{3} \sigma_{x}^{2} B}\langle S(x, y)\rangle \approx \frac{0.84\left(\frac{N_{\bar{p}}}{6 \times 10^{10}}\right)}{\sigma_{x}^{2}[\mathrm{~mm}] B[\mathrm{kG}]}\langle S(x, y)\rangle .
$$

Figure 12 shows the numerical factor $S(x, y)$ for the two electron distributions satisfying Eq. (35) with $a=\sigma_{x}$ (left-hand plot) and another with $a=2.5 \sigma_{x}$ (right-hand plot). The maximum value of this factor $S^{\max }(x, y)$ is 0.7 for the slender electron beam and 0.13 for the wider electron beam, and it occurs at amplitudes of about the electron beam size. The coupling vanishes for small and very large betatron amplitude particles. The effect is larger in the plane of the larger antiproton ellipse axis (horizontal in our case).

Consider a numerical example with the same parameters we used above: $\sigma_{x}=0.61 \mathrm{~mm}, N_{\bar{p}}=6 \times 10^{10}$, $\xi_{x}^{e} \simeq 0.01$. The maximum numerical factor in Eq. (42) is $\langle S(x, y)\rangle^{\max } \approx 0.5 S^{\max }(x, y)$, i.e., 0.35 for $a=1 \sigma_{x}$ and 0.065 for $a=2.5 \sigma_{x}$. With a solenoid field of $B=$ $40 \mathrm{kG}$, one gets the maximum coupling spread $|\kappa| \simeq$ $2 \times 10^{-4}$ for a thin electron beam, and $3.5 \times 10^{-5}$ for a wider electron beam. Both of these values are rather small with respect to the Tevatron global coupling correction

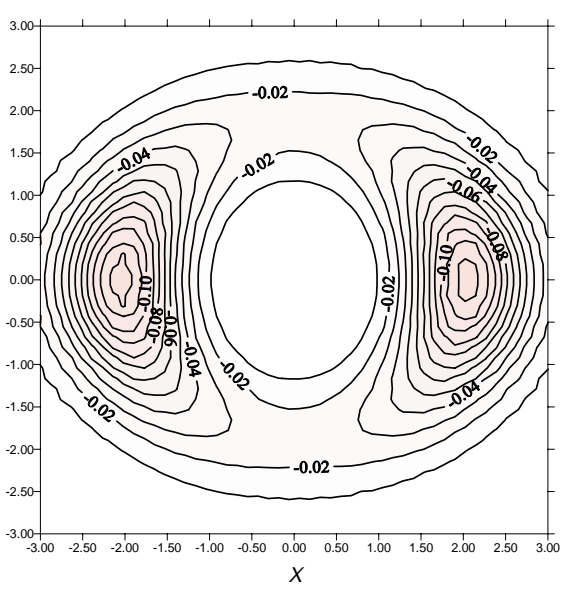

FIG. 12. (Color) Coupling functions $S(x, y)$ for antiproton betatron oscillations with thin (left) and wide electron beams (right). $x$ and $y$ coordinates are given in units of $\sigma_{x}$. 
goal of about 0.001. Note that two $2 \mathrm{~m}$ long $40 \mathrm{kG}$ solenoids for the electron lenses will contribute a static coupling of about $|\kappa| \simeq 0.001$.

\section{Head-tail effect due to electron beam}

This section is devoted to the stability of the antiproton beam interacting with an electron beam in the electron lens setup for beam-beam compensation in the Tevatron collider. Electron space-charge forces cause transverse head-tail coupling within the antiproton bunch which may lead to a transverse mode coupling instability (TMCI). A detailed theory, analytical studies, and numerical simulations of the effect can be found in Ref. [24]. Here we present estimates of the threshold longitudinal magnetic field necessary to avoid the instability and the dependence of the threshold on electron and antiproton beam parameters.

Low energy electrons can create significant transverse impedance comparable with the intrinsic impedance of the Tevatron ring, and this can result in a collective instability of the antiproton bunch. The electron beam is to be born on an electron gun cathode, transported through the interaction region, and absorbed in the collector. Therefore, each portion of electrons passes through the antiproton beam only once, and only short distance transverse wake fields are of interest. The phenomenon is as follows: If the centroid of the antiproton bunch head collides off the electron beam center, then the electronantiproton repulsion causes the electron motion. As a result, the electron beam has a displacement when it interacts with the tail of the bunch. Thus, the impact of the electron beam on the following antiprotons depends on the transverse coordinate of the preceding antiprotons. Such a head-tail interaction leads to the TMCI.

This effect is similar to the "strong head-tail" interaction via vacuum chamber impedance first observed a long time ago in electron storage rings [25]. The TMCI in the electron rings limits the maximum single bunch current. In our case, the source of the coupling is the electron space charge which is the basic mechanism for the beambeam compensation and, thus, cannot be avoided. The way to counteract the instability is to increase the electron beam rigidity, to make its motion during the collision smaller. Naturally it can be done using a strong longitudinal magnetic field in the interaction region ${ }^{2}$.

\section{Direct and skew wakes}

Conventionally, the analysis of relativistic beam stability relies on the wake function concept; see, e.g., [25].

\footnotetext{
${ }^{2}$ It is assumed that the Tevatron ring chromaticity can be made close to zero, so that the "weak head-tail" [25] instability is negligible.
}

Electromagnetic fields excited in an accelerator vacuum pipe vary over transverse distances of about the pipe aperture $b$, which is usually much larger than the beam radius $a$. That allows an expansion of the perturbation on dipole, quadrupole, and higher order terms over a small parameter $(a / b)$.

The situation is different for the case under study. The electron beam space-charge fields excited by antiprotons have about the same transverse extent as the antiproton beam, and this complicates the analysis. However, the interaction can be described by the conventional approach for a specific case when both the antiproton bunch and the electron beam are homogeneous and bounded by the same radius $a=a_{\bar{p}}$. In this case, electromagnetic wake fields have a simple radial structure and can be easily calculated.

To find the dipole wake function, let us consider a thin antiproton slice with a charge $q$ and offset $\Delta x$ traveling through the electron beam. After interacting with the slice, electrons acquire a transverse velocity given by

$$
v_{x e}=\frac{2 e q \Delta x}{a^{2}\left(1+\beta_{e}\right) \gamma_{e} m c} .
$$

Such a kick causes transverse Larmor oscillations in the longitudinal magnetic field $B$, and, after a time interval $t$, the electron transverse offsets are

$$
x_{e}=\frac{v_{x e}}{\omega_{L}} \sin \left(\omega_{L} t\right), \quad y_{e}=\frac{v_{x e}}{\omega_{L}}\left[1-\cos \left(\omega_{L} t\right)\right],
$$

where $\omega_{L}=e B /\left(\gamma_{e} m c\right)$ stands for the Larmor frequency. One can see that an originally horizontal displacement $\Delta x$ results in both horizontal and vertical displacements. Taking into account the possibility of a vertical offset $y$, we conclude that antiprotons at a distance $s$ behind the slice will experience momentum changes equal to

$$
\begin{aligned}
& \Delta p_{x}(s)=-\frac{e q}{c}\left[W_{d}(s) \Delta x-W_{s}(s) \Delta y\right], \\
& \left.\Delta p_{y}(s)=-\frac{e q}{c}\left[W_{s}(s) \Delta x+W_{d}(s) \Delta y\right)\right],
\end{aligned}
$$

where we introduce the direct wake function $W_{d}(s)$ and the skew $W_{s}(s)$ wake function

$$
W_{d}(s)=W \sin (k s), \quad W_{s}(s)=W[1-\cos (k s)],
$$

$$
\begin{aligned}
W_{d, s}(s) & =0, \text { if } s \leq 0, \text { and } \\
W & =\frac{4 \pi n_{e} L_{e}}{\left(1+\beta_{e}\right) a^{2}(B / e)}, \quad k=\frac{\omega_{L}}{\left(1+\beta_{e}\right) c},
\end{aligned}
$$

where $n_{e}=\frac{J_{e}}{e \pi a^{2} v_{e}}$.

Depending on the parameters, one or another of the two wake functions (46) can give a dominant influence on the antiproton beam stability. The direct wake effects are suppressed if there are many Larmor oscillations periods 
over the antiproton bunch length $\sigma_{s}$, while the skew force impact decreases with increasing $x-y$ detuning.

In the case of the Tevatron operating near the coupling resonance $\Delta \nu=\left|\nu_{x}-\nu_{y}\right| \approx 0.01$, consideration of the coupling of only the two closest modes $\nu_{x}+m \nu_{s}$ and $\nu_{y}+n \nu_{s}$ ( $m, n$ are integer) gives the following expression for the threshold magnetic field [24]:

$$
B_{\mathrm{thr}} \approx 1.3 \frac{e N_{\bar{p}} \sqrt{\xi_{x}^{e} \xi_{y}^{e}}}{a^{2} \sqrt{\Delta \nu \nu_{s}}} .
$$

For $\xi_{x}^{e}=\xi_{y}^{e}=-0.01, \quad N_{\bar{p}}=6 \times 10^{10}, \quad \nu_{s}=0.001$, $\Delta \nu=0.01, a=1 \mathrm{~mm}$, the solenoid magnetic field has to be more than $B_{\mathrm{thr}}=12 \mathrm{kG}$.

\section{Multimode analysis}

The two mode coupling model allows us to derive analytical formulas for the TMCI threshold by taking into account only a constant skew component of the wake force due to the electron beam and just two coupling modes. A more general numerical algorithm for calculating the mode coupling is developed in Ref. [26] and it avoids such simplifications and considers many modes and a general wake form, and, most importantly, it deals with nonaveraged motion. In that analysis, the antiproton bunch is divided into several radial and azimuthal parts in synchrotron phase space, and, consequently, a series of synchrobetatron modes can be seen. The wake force kick changes the backward particles' angles. The rest of the accelerator is presented by a linear transformation matrix (rotation in phase space). Eigenvalues (eigentunes) of the resulting transformation matrix can be calculated numerically. The complexity of the calculations is squared as the number of modes, so, for calculations with MATHCAD software one has to limit the number.

We divide the bunch into 1 radial (i.e., the same synchrotron oscillations amplitude for all particles) and 7 azimuthal parts for both vertical and horizontal degrees of freedom. Thus, it is possible to see the behavior of the first 1 radial and 7 azimuthal synchrobetatron modes in horizontal and vertical motion taking into account their coupling. Complete expressions for the linearized direct and skew transverse wake functions, Eq. (46), are used.

Numerical parameters used in these calculations are $N_{\bar{p}}=6 \times 10^{10}$, the rms size of the round Gaussian antiproton beam is $\sigma_{\bar{p}}=1 \mathrm{~mm}$, and the longitudinal magnetic field is equal to $10 \mathrm{kG}$. Figure 13 shows the eigentunes versus the linear betatron tune shift $\xi_{e}$ due to interaction with electron beam. The fractional part of the betatron tune for the horizontal motion is $\nu_{x}=0.556$ and for the vertical $\nu_{y}=0.555$, and the synchrotron tune is 0.001 . Therefore, the betatron tune difference is exactly the synchrotron tune. If $\xi_{e}=0$, then the eigenfrequencies of the azimuthal modes are equal to $\nu_{x, y}+k \nu_{s}$, where the integer $k$ has seven values in the range of $-3, \ldots, 3$ and represents the number of

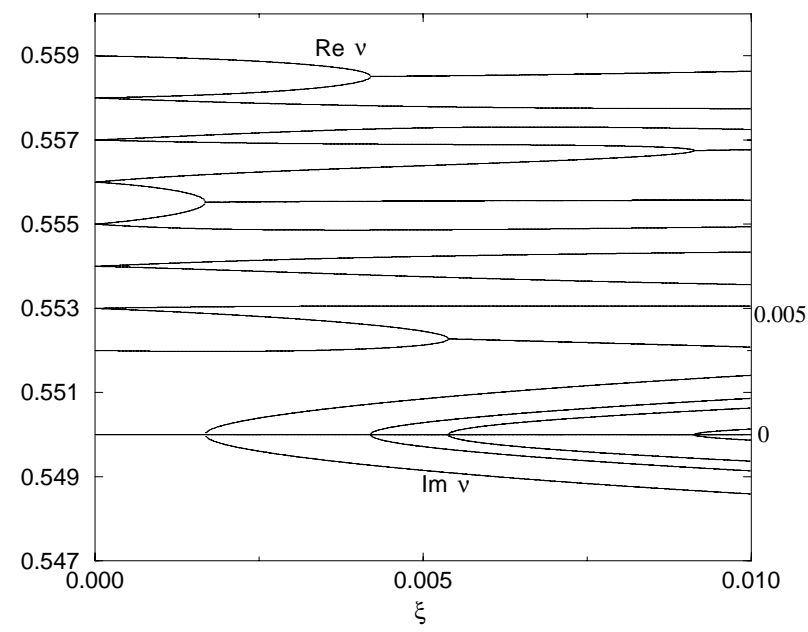

FIG. 13. Eigenfrequencies (tunes) of the antiproton bunch oscillation modes vs the antiproton betatron tune shift due to the electron beam $\xi^{e}$ (horizontal axis). The vertical scale on the left is for the fractional part of the tunes $\operatorname{Re} \nu$ (upper series of lines), and the right-side scale is for the imaginary part of the tunes $\operatorname{Im} \nu$ (lower series of lines).

modulation periods in synchrotron phase space. Some of the modes coalesce with increasing $\xi_{e}$, and the real parts of their tunes $\operatorname{Re} \nu$ (see upper series of curves in Fig. 13) become equal, while the imaginary parts $\operatorname{Im} \nu$ bifurcate into one negative and one positive branch. The latter evidently means instability in the motion. In our case, the first merging of modes takes place at $\xi \approx .0017$, the next merging of higher modes occurs at $\xi \approx .0045$, etc.

Figure 14 shows the tune shift threshold $\xi^{e}$ for the first coupling modes versus the tune split in units of the synchrotron tune $\Delta \nu=\left(\nu_{x}-\nu_{y}\right)$ for a vertical tune equal to 0.555 . The threshold grows linearly until

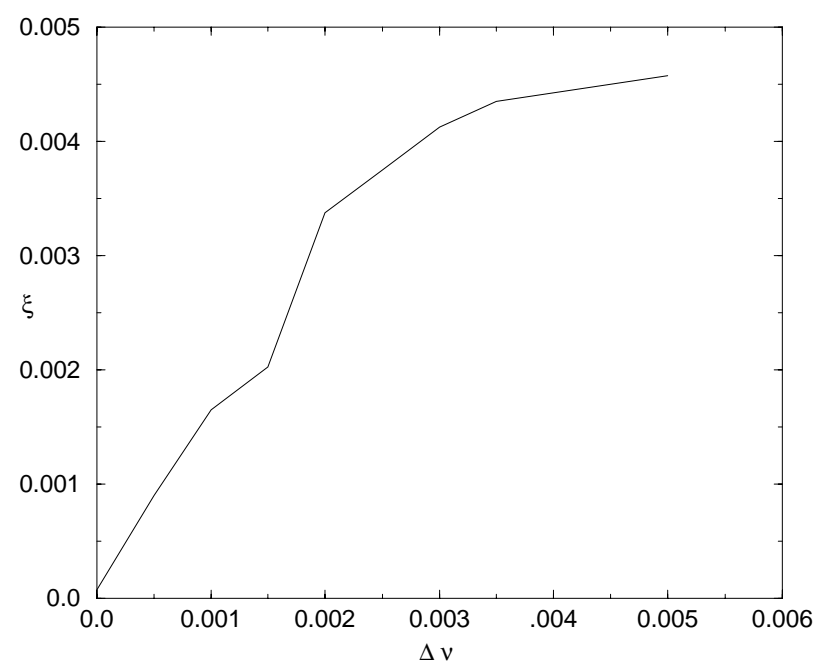

FIG. 14. Threshold antiproton tune shift $\xi^{e}$ (vertical axis) due to the electron beam vs the difference of antiproton horizontal and vertical tunes $\Delta \nu=\nu_{x}-\nu_{y} . \quad B=10 \mathrm{kG}, \nu_{s}=0.001$, $N_{\bar{p}}=6 \times 10^{10}$. 
$\Delta \nu \approx(2-2.5) \nu_{s}$ and then is approximately proportional to $\sqrt{\Delta \nu}$ - in good agreement with the two mode model. Note that a completely adequate consideration of the fast oscillating parts of the wakes would require many more modes $\sim k \sigma_{s} \simeq 30-100$ to be taken into account.

\section{TMCI simulations with electron beam}

Three-dimensional simulations of the effects have been done with a numerical code [24]. The round Gaussian antiproton beam $\left(\sigma_{x}=\sigma_{y}=\sigma_{\bar{p}}\right)$ is presented as a number of macroparticles (typically in the range from $M=128$ to maximum 2048). The particles have equal charges $e \Delta N_{\bar{p}}=e N_{\bar{p}} / M$. Both direct and skew wakes are taken into account in this numerical simulation. The simulation reveals that, although the antiproton bunch motion is essentially two-dimensional (since the wake is 2D), the instability starts in that plane where the original lattice tune is closer to half integer $\nu=1 / 2$, e.g., in the horizontal plane for the Tevatron ring.

Figure 15 shows results of the numerical simulations giving the threshold strength of the solenoidal magnetic field $B_{\mathrm{thr}}$ vs the electron beam intensity parameter $\xi^{e}$ for antiproton bunch populations equal to $N_{\bar{p}}=(1,6,10) \times$ $10^{10}$ - lower, middle, and upper curves, respectively. We define the threshold as the value of $B$ which results in more than a tenfold increase of the initial centroid betatron amplitude over the first 10000 turns. One can see that the field is approximately proportional to both $\xi^{e}$ and $N_{\bar{p}}$ in accordance with the theoretical prediction

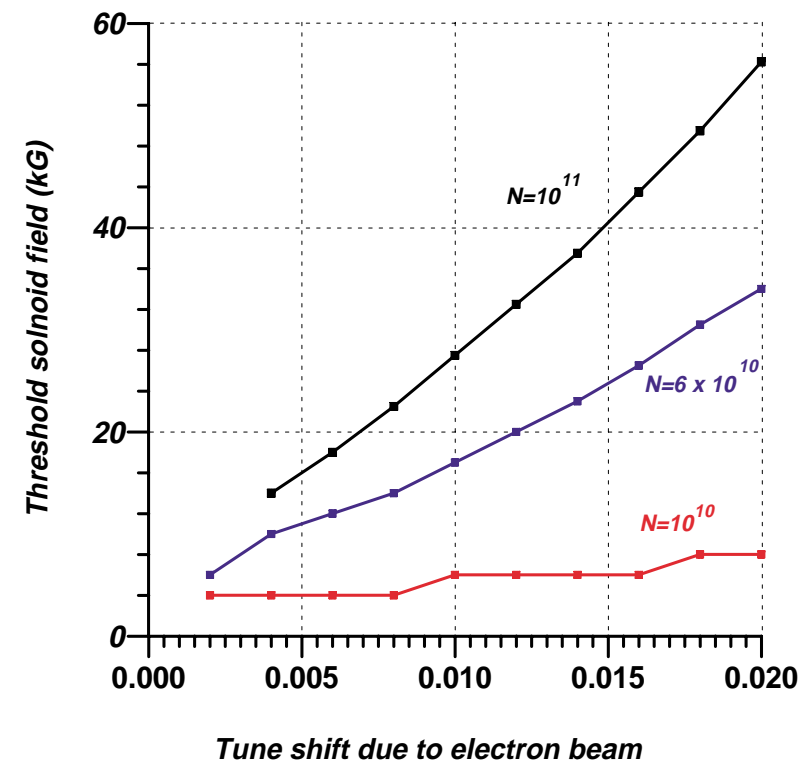

FIG. 15. (Color) Threshold solenoid field $B_{\text {thr }}$ vs tune shift due to electrons $\left|\xi_{e}\right|$ at different bunch populations $N_{\bar{p}}=$ $1,6,10 \times 10^{10}$. Focusing lattice tunes $\nu_{x}=0.585, \nu_{y}=$ 0.575 , synchrotron tune $\nu_{s}=0.0012$, no betatron tune spread in the beam, and the rms size of antiproton beam $\sigma_{\bar{p}}=$ $0.7 \mathrm{~mm}$.
Eq. (48). We found that the dependence of the threshold on the synchrotron tune agrees well with theory, also, i.e., $B_{\text {thr }} \propto \sqrt{\nu_{s}}$.

In order to evaluate the importance of the oscillation part of the wakes in Eq. (46), we performed similar simulations without the constant part of the skew wake, i.e., with $W_{d}(s)=W \sin (k s)$ and $W_{s}(s)=-W \cos (k s)$. We found that a solenoid field about 5 times smaller is required for stability. This confirms the decisive role of the constant part of the skew wake that is a basic assumption of the two-mode coupling model.

The TMCI threshold sensitively depends on the operation point $\nu_{x}, \nu_{y}$. Figure 16 presents the results of scanning the horizontal tune $\nu_{x}$ from 0.52 to 0.63 while the vertical tune is held constant at $\nu_{y}=0.575$. In close vicinity to the coupling resonance $\Delta \nu=\left|\nu_{x}-\nu_{y}\right| \leq$ $15 \nu_{s}$, the threshold magnetic field depends on $\nu_{s}$ approximately as $\propto 1 /|\Delta \nu|^{\kappa}$, where $2 / 5<\kappa<1 / 2$. Away from the resonance, the best fit power is $\kappa \approx 1 / 5$. The tune dependence on the tune split is different from Eq. (48) if $|\Delta \nu|$ is more than $15 \nu_{s} \approx 0.015$. The threshold also goes up near the half-integer resonance $\nu_{x} \rightarrow 0.5$.

In order to compare simulations with the two mode model, one can fit $B_{\text {thr }}$ in a form similar to Eq. (48)

$$
B_{\mathrm{thr}} \approx \frac{0.95 e N_{\bar{p}} \xi^{e}}{\sigma_{\bar{p}}^{2} \sqrt{\left|\nu_{x}-\nu_{y}\right| \nu_{s}}}=\frac{17.5[\mathrm{kG}] \frac{N_{\bar{p}}}{6 \times 10^{10}}\left|\frac{\xi^{e}}{0.01}\right|}{\left(\frac{\sigma_{\bar{p}}[\mathrm{~mm}]}{0.7}\right)^{2} \sqrt{\frac{\nu_{s}}{0.001} \frac{|\Delta \nu|}{0.01}}}
$$

see also the dashed line in Fig. 16. There are differences in numerical factors between Eqs. (49) and (48) because

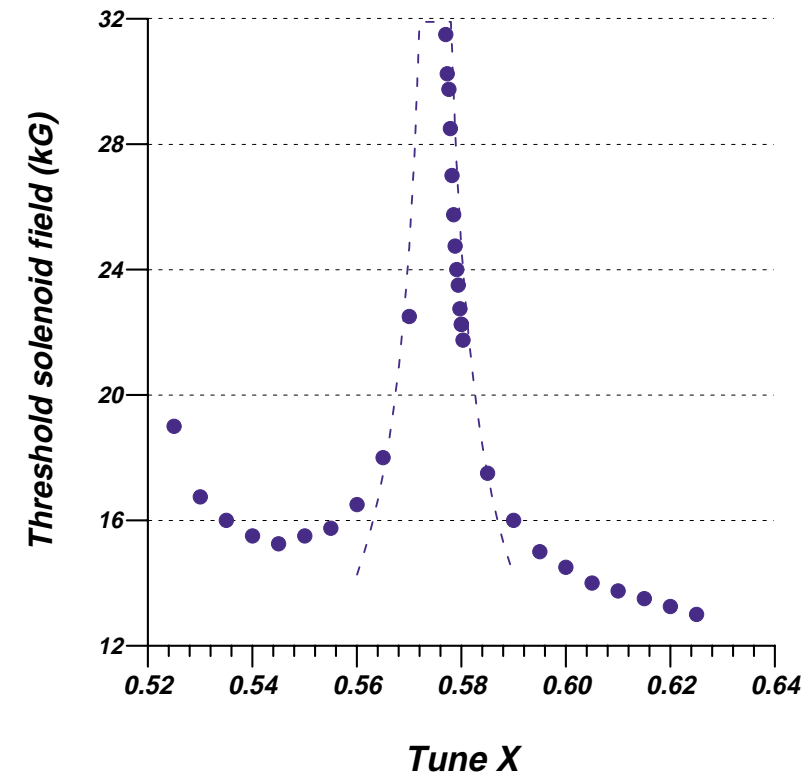

FIG. 16. (Color) Threshold magnetic field vs horizontal tune $\nu_{x}$. Dashed line corresponds to $B_{\mathrm{thr}} \propto 1 / \sqrt{\left|\nu_{x}-\nu_{y}\right|}$; $\nu_{y}=0.575, \quad \nu_{s}=0.001, \quad \xi^{e}=-0.01, \quad N_{\bar{p}}=6 \times 10^{10}$, $\sigma_{\bar{p}}=0.7 \mathrm{~mm}$. 
(a) the kick due to a Gaussian beam differs from a round beam kick Eq. (43) and $\sigma_{\bar{p}}$ is used instead of $a$, (b) the oscillating parts of the wake forces are taken into account in the simulations in contrast to the two mode model, and (c) more than two modes play a role in the computer tracking because of the large number of macroparticles. Nevertheless, there is excellent quantitative agreement with the results of the multimode analysis presented in Fig. 14.

Neither the two-mode theory nor the multimode analysis deal with the tune spread in the antiproton bunch, which will tend to suppress the instability due to Landau damping [27]. The TMCI can be additionally suppressed if the electron beam has a radius larger than the antiproton beam radius, $a \geq \sigma_{\bar{p}}$. It was found out in Ref. [24] that the skew wake function scales with the electron beam radius as $W_{s} \propto n_{e} / a^{2}$; i.e., using a 2 times wider electron beam will lead to 4 times smaller required magnetic field for the same $\xi^{e}$. The oscillating direct wake function $W_{d}(s)$ does not depend on the electron beam radius when the electron density is fixed. Coupling of higher than dipole single bunch modes is the subject for further studies.

\section{Effect on proton beam, long-term control}

Since the Tevatron collider operates with many more protons per bunch than antiprotons per bunch, the antiproton beam-beam tune shift is larger than the proton tune shift. Therefore, the compensation will be necessary only for antiprotons. The direction of the electrons' propagation is opposite to the antiprotons velocity (i.e. they collide ${ }^{3}$ ). The proton beam moves in the opposite direction in the same vacuum chamber and also may effectively interact with the electron current. If the proton and antiproton beam orbits are not separated, an additional positive tune shift for protons is

$$
\xi_{p}^{e} \approx \frac{N_{e} r_{p}\left(1-\beta_{e}\right)}{4 \pi \varepsilon_{n}}=\xi^{e} \frac{1-\beta_{e}}{1+\beta_{e}} .
$$

If $\beta_{e} \ll 1, \xi_{p}^{e}$ does not differ too much from $\xi^{e} \simeq 0.01$ which is supposed to be too large to tolerate. One needs to avoid this impact on the proton beam due to electron charge. Separation of the proton and electron beams can help a lot; e.g., a separation of $d \approx 10 \sigma_{p} \simeq 7 \mathrm{~mm}$ causes quite a minor proton beam tune shift of about

$$
\xi_{p}^{e}(d) \approx \frac{2 \xi^{e}}{\left(d / \sigma_{p}\right)^{2}} \frac{1-\beta_{e}}{1+\beta_{e}} \sim 1.3 \times 10^{-4},
$$

with vertical and horizontal having opposite signs.

A very important issue is longer term control of the electron beam. The amount of required tune spread

\footnotetext{
${ }^{3}$ Because of the very small inelastic $e-\bar{p}$ cross section, the antiproton beam lifetime does not depend significantly on electron current.
}

compensation varies in time, e.g., at injection proton and antiproton beams are everywhere separated and that yields one pattern of the footprint; after acceleration beams collide at two IPs, the separator strength goes down, and they provide different separations at parasitic crossings, etc., and, consequently, the tune footprint is changed; finally, after a few hours the intensity and emittances of the beams are significantly different. The electron lens has to be adjusted in order to compress the tune area effectively. One way to reach the goal is to rely on a beam-beam model which predicts the footprint from measured data on bunch intensities, emittances, and separation. Another choice is to continuously measure the beams' tune spectra and make necessary corrections in electron beam current, size, and distribution (i.e., implement a kind of long-term feedback). Reliability of the setup during collider operation may probably require multiple cathodes or guns.

\section{E. Electron current fluctuations}

Fluctuations of the electron current from turn to turn cause time variable quadrupole kicks which lead to a transverse emittance growth of the antiproton bunches. The current in the electron lens setup has to be modulated rather fast although periodically and, thus, the issue of how stable the current is at a one-turn scale may be of importance.

The emittance growth rate due to fluctuations of a gradient $\delta G$ of a lens with length $L$ is given by [28]

$$
\frac{d \varepsilon_{z}}{d t}=f_{0}^{2} \frac{\varepsilon_{z}}{16}\left(\frac{e L \beta_{z}}{P c}\right)^{2} \sum_{n=-\infty}^{\infty} S_{\delta G}\left(f_{0}\left|2 \nu_{z}-n\right|\right)
$$

where $f_{0}$ is the revolution frequency, $\beta_{0}$ is beta function at the lens location, $P$ is the antiproton momentum, $\nu_{z}$ is the machine tune, and $S_{\delta G}(f)$ is the power spectral density (PSD) of the gradient fluctuations. The PSD we use is defined for positive frequencies $f$. One can see that only some particular frequencies contribute to the emittance growth, and the lowest of them $\left(2 \Delta \nu_{z}-1\right) f_{0}$ is about $7.2 \mathrm{kHz}$ for the Tevatron. If one assumes that the current ripple is "white noise" with a constant PSD $S_{\delta G}$, then the rms value of the ripple $\delta G$ relates to the PSD as

$$
\delta G^{2}=(1 / 2) f_{0} S_{\delta G},
$$

and, therefore, taking into account that there are two electron beams (with presumably uncorrelated current fluctuations) on the antiproton orbit, one gets

$$
\frac{d \varepsilon_{z}}{d t}=2 \pi^{2} f_{0} \varepsilon_{z}\left[\left(\xi_{1 z}^{e}\right)^{2}+\left(\xi_{2 z}^{e}\right)^{2}\right]\left(\frac{\delta J}{J}\right)^{2},
$$

where $F_{1,2}$ are focal lengths of the two electron beams, $\delta J / J$ is the rms value of relative current fluctuation, $\xi_{1,2, z}$ are the values of tune shift produced by electron lenses 1 and 2 , respectively (proportional to the current), and we used the relation $\xi^{e}=(1 / 4 \pi) \beta_{z} / F$. 
From Eq. (53) one immediately gets the emittance evolution equation

$$
\varepsilon_{z}(t)=\varepsilon_{0 z} \exp \left(t / \tau_{z}^{e}\right),
$$

where the characteristic growth time is equal to

$$
\tau_{z}^{e}=\frac{1}{4 \pi^{2} f_{0}\left[\left(\xi_{1 z}^{e}\right)^{2}+\left(\xi_{2 z}\right)^{2}\right]\left(\frac{\delta J}{J}\right)^{2}} .
$$

The growth time is different for different bunches; e.g., $\tau_{z}^{e}$ is smaller for the bunches which experience larger currents $J$ and, therefore, tune shifts $\xi^{e}$. These bunches (named PACMAN bunches) are usually located near the gaps (see Fig. 4). Let us take, for example, one of those bunches with $\xi_{1 x}^{e}=0.01$ and $\xi_{2 x}^{e}=0.002$, then a requirement of $\tau_{x}^{e}>10 \mathrm{~h}$ results in $\frac{\delta J}{J}<0.53 \times 10^{-3}$. If one assumes a constant distribution function for the ripple $^{4}$, then the value above corresponds to peak-to-peak current fluctuations of

$$
\frac{\Delta J}{J} \approx 1.8 \times 10^{-3} \text {. }
$$

For non-PACMAN bunches (inside the batch, away from the gaps), the requirement is somewhat less stringent $\frac{\Delta J}{J}<3.2 \times 10^{-3}$. This current stability will require a stable voltage $U_{e}$ of the (modulated) power supply which controls the electron gun current ${ }^{5}$ of $\left(\frac{\delta U_{e}}{U_{e}}\right)=\frac{2}{3}\left(\frac{\delta J}{J}\right)<$ $10^{-3}$.

\section{F. Transverse electron beam motion}

Transverse motion of the electron beam may also cause direct antiproton emittance growth. Indeed, if the electron beam displacement is equal to $\delta X$, then the dipole kick experienced by antiprotons is $\delta \theta=\delta X / F$, where $F$ is the focal length of the defocusing electron lens. Coherent antiproton betatron oscillations occur and after some decoherence time they result in antiproton emittance growth. The normalized emittance grows linearly in time and its growth rate is equal to [29]

$$
\frac{d \varepsilon_{x}}{d t}=\frac{\gamma_{\bar{p}} f_{0}^{2}}{4} \sum_{\text {sources }} \frac{\beta_{x}}{F^{2}} \sum_{n=-\infty}^{\infty} S_{\delta X}\left(f_{0}\left|\nu_{x}-n\right|\right) .
$$

Note that the frequencies of interest $f_{0}\left|\nu_{x}-n\right|$ start from the betatron frequency of the Tevatron $\left(1-\Delta \nu_{x}\right) f_{0} \approx 20 \mathrm{kHz}$.

Using the same transformations as above, one gets for two electron lenses

$$
\frac{d \varepsilon_{x}}{d t}=8 \gamma_{\bar{p}} \pi^{2} f_{0} \delta X^{2}\left(\frac{\left(\xi_{1 x}^{e}\right)^{2}}{\beta_{1 x}}+\frac{\left(\xi_{2 x}^{e}\right)^{2}}{\beta_{2 x}}\right),
$$

where $\delta X$ now stands for the rms electron beam vibration amplitude.

\footnotetext{
${ }^{4}$ For such a distribution the rms value is $1 / \sqrt{12}$ of the peakto-peak value.

${ }^{5}$ Taking into account Child's law (10).
}

Let us constrain the emittance growth rate to be less than $\varepsilon_{n} / 10 \mathrm{~h}, \varepsilon_{n}=2.5 \pi \mathrm{mm}$ mrad. Then, for the PACMAN bunches we get the following requirement on the rms electron beam turn-to-turn position stability:

$$
\delta X \leq 0.14 \mu \mathrm{m} .
$$

For the bunches in the middle of the bunch train, the requirement is about $0.21 \mu \mathrm{m}$.

The tolerable amplitudes are several orders of magnitude larger than vibrations of the Tevatron quadrupoles at high frequencies. Accordingly to [30], the rms amplitude of the Tevatron quadrupole magnets at frequencies above $100 \mathrm{~Hz}$ is about $2 \mathrm{~nm}$, and the amplitude rapidly goes down with increase of frequency. However, other sources of electron beam jitter have to be checked.

If the electron beam and the antiproton beam are not properly aligned with respect to each other and they collide off center with displacement equal to $\Delta X$, then electron current ripple at betatron frequencies causes dipole kicks on antiprotons and can also lead to transverse emittance growth. The tolerance can be easily estimated from Eq. (59) as

$$
\frac{\Delta J}{J} \Delta X \approx \delta X .
$$

Note that the tolerance depends on the straightness of the electron beam in the interaction region, which is determined by the solenoid field quality. Using $\Delta X=$ $0.25 \sigma_{\bar{p}}=0.15 \mathrm{~mm}$, one calculates the rms current ripple tolerance for the PACMAN bunches as $\frac{\Delta J}{J}<1.1 \times$ $10^{-3}$, or about $0.37 \%$ peak-to-peak, and about $1.6 \times$ $10^{-3}$ the rms value and $0.52 \%$ peak-to-peak for nonPACMAN bunches. These requirements are somewhat loose in comparison with the quadrupole kick effect.

\section{G. Solenoid field quality}

A strong solenoid magnetic field $B$ of the order of several Tesla in the straight section of the electron lens assures that the electrons perform very small but fast Larmor oscillations around the magnetic field lines. Therefore, deviations of $\vec{B}$ from a straight line will cause off-center collisions of the antiproton and electron beams. In the case of the nonlinear electron lens this may cause unwanted nonlinear components of the forces. To avoid the effect, one needs to have the field lines not deviate from the straight antiproton orbit more than some part of the transverse antiproton beam size $\sigma_{\bar{p}} \approx 0.8 \mathrm{~mm}$. If one requires a solenoid field straightness equivalent to $\Delta X=0.2 \mathrm{~mm}$, then a transverse field component has to be less than

$$
\frac{\Delta B_{\perp}}{B} \sim \frac{\Delta X}{L} \sim \frac{0.2 \mathrm{~mm}}{2 \mathrm{~m}}=10^{-4} .
$$

This is comparable with the field quality in numerous electron cooling devices. 


\section{DISCUSSION AND CONCLUSIONS}

\section{A. Historical overview}

The idea of the compensation of the beam-beam interaction has been discussed previously for other collider facilities, mostly $e^{+} e^{-}$(one of the earliest publication is Ref. [31]). The theory of compensated electron-positron collisions in storage rings [32] predicts that collective instabilities in the circulating beams limit the performance and do not allow significant benefits with respect to the usual uncompensated case. Experiments with compensated $e^{+} e^{-}$beams were carried out at the DCI collider at the Laboratoire de l'Accelerateur Lineaire (Orsay, France) in the 1970s [33]. There were two intersecting rings with four equally populated beams (positrons and electrons in each ring) which collided at the same point. This arrangement yielded a space charge and a current compensation factor of about 5-10. It allowed an increase of the maximum beam-beam parameter $\xi$ from 0.018 to 0.024 . Nevertheless, there was no significant increase in luminosity, and it was concluded that the value of $\xi$ rather than the residual compensated value of $\xi_{r}=\xi /(5-10)$ sets the limit. Stability regions, smaller in size than those observed in the two-beam configuration, were found to decrease rapidly with current, probably because of collective modes. A decade later, experimental studies at VEPP- $4 e^{+}-e^{-}$collider demonstrated that partial compensation of the cubic nonlinearity in the beam-beam interaction with use of octupole lenses may result in a reduction of particle loss rate and a gain in the machine efficiency [34]. Similar effects are predicted in a recent theoretical analysis [35].

In linear $e^{+} e^{-}$colliders, the beams collide once, and, therefore, there is no long-term memory in an opposing beam as in storage rings. Thus, collective phenomena are weaker. Charge separation in neutralized beams occurs only if the space-charge parameter is very large $\xi \gg 1$ $[36,37]$.

It was pointed out in Ref. [38] that compensation of beam-beam effects with an electron beam leads to elongation of the transverse decoherence time due to the smaller tune spread. This also leads to less stringent requirements on the feedback system for emittance preservation in large colliders like the Large Hadron Collider.

\section{B. Conclusions}

We have described a technique to compensate the beam-beam induced tune shift and tune spread of antiprotons in the proton-antiproton Tevatron collider with the use of low-energy high current electron beam. Implementations of the technique can include (1) the electron lens with modulated current which provides different linear defocusing forces for different antiproton bunches and, therefore, equalizes their betatron frequencies, (2) the electron compressor, a nonlinear but dc electron lens to compensate (on average) the nonlinear focusing of an- tiprotons due to the proton beam and, thus, to shrink the beam-beam footprint, and (3) a several times more powerful electron compressor to eliminate the crossing angle, and, therefore, to double the luminosity, or to allow more proton-antiproton interaction points, or to get the same luminosity with smaller beam intensities.

The electron beam setup looks much like an electron cooler, except electrons collide with antiprotons. The proton beam has to be separated from the other two. Electron beams $2 \mathrm{~m}$ long, $2 \mathrm{~mm}$ diameter, $10 \mathrm{kV}$ with $1-2 \mathrm{~A}$ of current are to be installed in places with large beta functions $(\sim 100 \mathrm{~m})$, away from the main interaction points (IPs - B0 and D0 at the Tevatron). A strong longitudinal magnetic field plays a significant role in maintaining stability of both electron and antiproton beams as well as in keeping the electron beam current distribution distortions, and, therefore, distortions of electron space-charge forces, within acceptable limits.

Reduction of the bunch-to-bunch tune variation can be done with two round electron beams with specially programmed time-variable electron currents. One of the electron lens setups has to be installed at a location where the vertical beta function is larger than the horizontal one $\beta_{y}>\beta_{x}$ and, therefore, will affect mostly the vertical antiproton tune; another requires the opposite relation $\beta_{y}<\beta_{y}$ for mostly horizontal tune changes. For better linearity of the electron lens, the size of the electron beam should be 2-3 times the rms size of the antiproton beam. The electron current needed is periodic with the Tevatron revolution frequency. The current waveform (amplitude and modulation) depends on the particular colliding bunches pattern, bunch intensities, crossing angle, and orbit separation. We considered the time structure of the defocusing force due to electron current and estimated that the $132 \mathrm{~ns}$ bunch spacing in TEV33 will require 100-120 ns current modulation time in the $2 \mathrm{~m}$ long $10 \mathrm{kV}$ electron lenses.

Electron current fluctuations from turn-to-turn (more precisely, at frequencies about double the betatron frequency) should be less than $\Delta J_{e} / J_{e}<(2-3) \times 10^{-3}$ peak-to-peak. Otherwise variable defocusing kicks may lead to significant transverse antiproton emittance growth. The transverse emittance growth caused by the dipole kick due to a displaced electron beam is a less stringent requirement on the current ripple, depending on how well the electron and the antiproton beams are centered at the interaction region. Direct emittance growth with ideally centered beams due to electron beam vibrations is predicted to be negligible.

Nonlinear beam-beam compensation and the footprint compression require precise control of the electron beam shape. In principle, that can be done with use of near cathode electrodes in the diode electron gun.

We have considered distortions of the electron beam in the beam-beam compensation setup. It is found that a rather low longitudinal field of about $1 \mathrm{kG}$ can avoid the 
beam blow-up due to defocusing electron and antiproton space-charge forces. A much higher solenoid field of about $20-40 \mathrm{kG}$ is necessary to have electron charge distribution distortions within a few percent with respect to the original axisymmetric distribution. The need comes from a requirement to contribute much less $x-y$ coupling than other sources in the Tevatron collider ring, and not to introduce significant spread of the coupling in the antiproton bunch. Analytical considerations have shown that the distortion is smaller if the electron beam size is several times the antiproton beam size.

We have considered head-tail coupling in the Tevatron antiproton beam due to the electron beam impedance. The coupling can cause a single antiproton bunch instability if the magnetic field is less than a threshold value of the order of $20 \mathrm{kG}$. Consequently, to assure stability, a 40-60 kG longitudinal magnetic field has to be in the interaction region of the electron lens. A two-mode model agrees with multimode analysis and numerical simulations results.

The general conclusion is that beam-beam compensation with an electron beam looks very promising and it provides additional powerful knobs to control beam dynamics in the Tevatron collider. We find no severe requirements on the electron beam for the suggested device, and believe that realization of the idea will give benefits for the Tevatron.

\section{Experimental test}

An experimental installation that should demonstrate the feasibility of the electron lens is now under construction at Fermilab. This setup will serve as a prototype of the device that can later be inserted into the Tevatron ring.

The goals of the setup are to obtain a $10 \mathrm{kV}, 2 \mathrm{~m}$ long electron beam with total current up to $2 \mathrm{~A}$ propagating in a precise solenoid magnet, to test the current modulation in a few $\mathrm{MHz}$ bandwidth, and to study the beam dynamics. The parameters of the experimental installation are about the same as for the full scale device, except with a somewhat lower magnetic field and current density. The setup will allow study of the measures to suppress the two beam drift instability and testing of all the physical and technical solutions needed to build the electron lens for beam-beam compensation in the Tevatron.

The experimental installation consists of a diode electron gun and collector immersed in a magnetic field of $1-2.5 \mathrm{kG}$ produced by $0.5 \mathrm{~m}$ long normal conducting solenoids, and a $2 \mathrm{~m}$ long beam transport section inside a $4 \mathrm{kG}$ solenoid magnet. The beam radius at the cathode is $5 \mathrm{~mm}$ and it can be compressed to about 2.5 $\mathrm{mm}$ in the longer solenoid. The electron gun has special control electrodes to change the beam profile. The main solenoid of the installation is made precise enough so that the achievable angular field homogeneity is better than $5 \times 10^{-5}$. The magnetic field straightness will be measured optically, using a mirror with a magnetic arrow attached, and then improved, if necessary, by corrector coils.

\section{ACKNOWLEDGMENT}

We acknowledge helpful discussions on the subject of the beam-beam compensation with A. Sharapa, J. Marriner, L. Michelotti, J. Johnstone, and Y. Derbenev. We are thankful to S. Nagaitsev, I. Meshkov, and A. Shemyakin for many useful comments on electron beam stability and to W. Chou and D. Ritson for raising the question of electron beam position stability. We are indebted to A. Zinchenko and A. Burov for their collaboration in studies of the electron beam distortions and the TMCI due to the electron beam. Our special thanks to V. Parkhomchuk for attracting our attention to the electron beam impedance effect and to P. Bagley for provision of the multibunch tune simulations data. We acknowledge discussions on various aspects of the compensation with attendants of the Mini-Workshop on Beam-Beam Compensation (February, 1998, Fermilab). FNAL is operated by Universities Research Association, Inc., under Contract No. DE-AC02-76CH00300 with the U.S. Department of Energy.

[1] J. P. Marriner, in Proceedings of the 1998 European Particle Accelerator Conference, Stockholm, Sweden (Institute of Physics, Bristol, UK, 1998), p. 8.

[2] P. Bagley et al., Report No. FERMILAB-Conf-96/392, 1996.

[3] G. Dugan, in Proceedings of the 1989 IEEE Particle Accelerator Conference, Chicago, Illinois (IEEE, Piscataway, NJ, 1989), p. 426.

[4] D. Finley, in Proceedings of the III Advanced ICFA Beam Dynamics Workshop, Novosibirsk, 1989 (Budker INP, Novosibirsk, 1989), p. 34.

[5] D. Siergiej, D. Finley, and W. Herr, Phys. Rev. E 55, 3521 (1997).

[6] V. Shiltsev, Report No. FERMILAB-FN-653, 1997.

[7] V. Shiltsev and D. Finley, Report No. FERMILAB-TM2008, 1997.

[8] V. Shiltsev, Report No. FERMILAB-TM-2031, 1997.

[9] V. Danilov and V. Shiltsev, Report No. FNAL-FN-671, 1998.

[10] G. I. Budker et al., Part. Accel. 7, 197 (1976).

[11] J. R. Pierce, Theory and Design of Electron Beams (Van Nostrand, New York, 1954).

[12] A. Sharapa, A. Grudiev, D. Myakishev, and A. Shemyakin, Nucl. Instrum. Methods Phys. Res., Sect A 406, 169 (1998).

[13] L. P. Smith and P. L. Hartman, J. Appl. Phys. 2, 320 (1940).

[14] J.D. Lawson, The Physics of Charged-Particles Beams (Clarendon Press, Oxford, 1988), 2nd ed.

[15] N. Dikansky, S. Nagaitsev, and V. Parkhomchuk, Report No. FNAL-TM-1998, 1997. 
[16] V. Shiltsev and A. Zinchenko, Phys. Rev. ST Accel. Beams 1, 064001 (1998).

[17] G. Kuznetsov, Nucl. Instrum. Methods Phys. Res., Sect. A 340, 204 (1994).

[18] See, e.g., I. N. Meshkov, Transportation of Intense Beams of Charged Particles (Budker INP, Novosibirsk, 1989) (in Russian).

[19] A. V. Burov, V. Kudelainen, V. Lebedev, V. Parkhomchuk, A. Sery, and V. Shiltsev, Report No. INP 89-116 1989 (in Russian); Report No. CERN/PS 93-03 (AR), 1993.

[20] A. V. Burov, Report No. INP 88-124, 1988.

[21] V.I. Kudelainen, V. Parkhomchuk, and D. Pestrikov, Zh. Tekh. Fiz. 53, 870 (1983) [Sov. Phys.-Tech. Phys. 28, 556 (1983)].

[22] J.E. Augustin, SLAC Note No. PEP-63, 1973; B.W. Montague, CERN Report No. CERN/ISR-GS/75-36, 1975.

[23] P. Bagley and J. Annala (private communication).

[24] A. Burov, V. Danilov, and V. Shiltsev, Phys. Rev. E 59, 3605 (1999); Report No. FNAL-Pub-98/195, 1998.

[25] A. Chao, Physics of Collective Beam Instabilities in High Energy Accelerators (Wiley, New York, 1993).

[26] V. V. Danilov and E. A. Perevedentsev, Nucl. Instrum. Methods Phys. Res., Sect. A 391, 77 (1997).

[27] D. V. Pestrikov, Nucl. Instrum. Methods Phys. Res., Sect. A 373, 179 (1996).

[28] G. V. Stupakov, Report No. SSCL-575, 1992.

[29] V. Lebedev, V. Parkhomchuk, V. Shiltsev, and G. Stupakov, Part. Accel. 44, 147 (1994).

[30] B. Baklakov et al., Phys. Rev. ST Accel. Beams 1, 031001 (1998).
[31] J.E. Augustin et al., in Proceedings of the 7th International Conference on High Energy Accelerators, Yerevan, 1969 (Publishing House of the Academy of Sciences of Armenian SSR, Yerevan, 1970), Vol. 2, p. 113.

[32] Ya. Derbenev, Report No. SLAC-Trans-0115, 1973; INP Report No. 70-72, 1970.

[33] J. Le Duff, M. P. Level, P. C. Martin, E. M. Sommer, and H. Zyngier, in Proceedings of the 11th International Conference on High Energy Accelerators, Geneva (Birkhauser Verlag, Basel, Boston, Stuttgart, 1980), p. 707; see also IEEE Trans. Nucl. Sci. 26, 3559 (1979).

[34] A. B. Temnykh, in Proceedings of the III Advanced ICFA Beam Dynamics Workshop, Novosibirsk, 1989 (Budker INP, Novosibirsk, 1989), p. 5.

[35] Y. K. Batigin and T. Katayama, Report No. RIKEN-AFAC-3, 1997.

[36] N. A. Solyak, Report No. INP 88-44, 1988; in Proceedings of the 13th International Conference on High Energy Accelerators, Novosibirsk (Budker INP, Novosibirsk, 1986), Vol. 1, p. 151.

[37] D. Whittum and R. Siemann, in Proceedings of the 1997 IEEE Particle Accelerator Conference, Vancouver, Canada (IEEE, Piscataway, NJ, 1998), p. 602.

[38] E. Tsyganov, A. Taratin, and A. Zinchenko, Fiz. Elem. Chastits At. Yad. 27, 675 (1996) [Phys. Part. Nuclei 27, 279 (1996)]; see also SSCL-Report No. 519, 1993; CERNSL/Note No. 95-116/AP, 1995; Report No. JINR-E9-96-4, 1996. 